\title{
Geochemistry of sandstones from the Miocene Surma Group, Bengal Basin, Bangladesh: Implications for Provenance, tectonic setting and weathering
}

\author{
M. JULleh JALALUR RAHMAN ${ }^{1 *}$ and ShigeYUKI SUZUKI ${ }^{2}$ \\ ${ }^{1}$ Department of Geological Sciences, Jahangirnagar University, Saver, Dhaka, Bangladesh \\ ${ }^{2}$ Department of Earth Sciences, Okayama University, Okayama 700-8530, Japan
}

(Received November 2, 2006; Accepted July 31, 2007)

\begin{abstract}
Twenty six sandstone samples from six wells penetrating the Miocene Surma Group in the Bengal Basin, Bangladesh, were analyzed by lithium metaborate/tetraborate fusion Inductively Coupled Plasma (ICP) and Inductively Coupled Plasma Mass Spectrometry (ICP-MS) and by petrographic microscope. The framework grains of the Surma Group sandstones are rich in quartz followed by lithic grains, feldspar and mica (predominantly white mica). The sandstones are dominantly quartzolithic and quartzose one in composition with abundant low-grade metamorphic, sedimentary lithics, low feldspars and little volcanic detritus, indicating that the sands were derived from a quartzose recycled orogen province.

The Surma Group sandstones have moderate to high $\mathrm{SiO}_{2}$ contents $(64-85 \%$; on average $77 \%)$, $\mathrm{TiO}_{2}$ concentrations averaging $0.5 \%, \mathrm{Al}_{2} \mathrm{O}_{3}$ contents of about $8.4 \%$, and $\mathrm{Fe}_{2} \mathrm{O}_{3}$ (total $\mathrm{Fe}$ as $\mathrm{Fe}_{2} \mathrm{O}_{3}$ ) $+\mathrm{MgO}$ content of around $4.5 \%$. Compared to the average sandstone value, the Surma Group sandstones are depleted in $\mathrm{CaO}(2.36 \%)$ and enriched in $\mathrm{Al}_{2} \mathrm{O}_{3}, \mathrm{Fe}_{2} \mathrm{O}_{3}$ and $\mathrm{Na}_{2} \mathrm{O}$. Geochemically, the sandstones are classified mainly as litharenites. The Chemical Index of Alteration (CIA) values for the Miocene Surma Group sandstones vary from 53 to 65 with an average of 59, indicating low to moderate weathering of the source areas. The average CIA value (59) is a little above than that (50) of the upper continental crust. The shales from the Miocene Surma Group show higher CIA ( 70-78) values, indicating significant weathering in the source. The geochemical characteristics suggest an active continental margin to passive margin setting for the Surma Group sandstones, and preserve the signatures of a recycled provenance. The $\mathrm{Eu} / \mathrm{Eu} *(\sim 0.69),(\mathrm{La} / \mathrm{Lu}) \mathrm{cn}(\sim 10.07), \mathrm{La} / \mathrm{Sc}$ ( 3.98), Th/Sc $(\sim 1.44), \mathrm{La} / \mathrm{Co}(\sim 3.84), \mathrm{Th} / \mathrm{Co}(\sim 1.40)$, and $\mathrm{Cr} / \mathrm{Th}(\sim 9.48)$ ratios as well as chondrite-normalized REE patterns with flat HREE, LREE enrichment, and negative Eu anomalies indicate derivation of the Surma Group sandstones from felsic rock sources.
\end{abstract}

Keywords: Miocene Surma Group sandstones, Bengal Basin, geochemistry, provenance, tectonic setting

\section{INTRODUCTION}

This paper describes the geochemical composition of Miocene sandstones from the Surma Group of the Bengal Basin (Fig. 1) based on 26 core samples collected from six petroleum exploration wells: Bakhrabad-10 (BK), Fenchuganj-2 (FG), Kailas Tila-2 (KT), Titas-11 (TT), Shalda Nadi-1 (SN), and Shahbazpur-1 (SB) (Fig. 1). The Surma sandstones used in this study are encountered at depth ranges between $1571 \mathrm{~m}$ and $4249 \mathrm{~m}$. Clastic sediments of the Surma Group have a thickness of 5000 $\mathrm{m}$. The sedimentary succession comprises subequal proportions of alternating sandstones and shales. The sandstone framework components of the subsurface Surma Group are quartzolithic and quartzose, with abundant sedimentary and low-grade metamorphic grains, but lesser amounts of volcanic constituents (Rahman, 1999). These

*Corresponding author (e-mail: jjrahman65@yahoo.com)

Copyright $@ 2007$ by The Geochemical Society of Japan. compositions are diagnostic of a quartzose recycled orogen province (sensu Dickinson, 1985). The geochemistry of the sandstones is of similar importance, because such studies can give information about the provenance, tectonic setting and weathering history of the source rocks. The geochemical composition of terrigenous sedimentary rocks is a function of the complex interplay of various variables, such as provenance, weathering, transportation and diagenesis (Bhatia, 1983). Recent investigations on geochemical characteristics of ancient and modern detritus have been carried out in order to infer the source rocks, provenance and tectonic setting (e.g., Potter, 1978; Bhatia, 1983; Hiscott, 1984; Bhatia and Crook, 1986; Roser and Korsch, 1986, 1988; Dabard, 1990; McCann, 1991; McLennan et al., 1993; Garver et al., 1996; Nesbitt and Young, 1996; Hayashi et al., 1997). The proposed major elemental geochemical parameters after Bhatia (1983), such as $\mathrm{Fe}_{2} \mathrm{O}_{3}+\mathrm{MgO} \%, \mathrm{TiO}_{2} \%$, $\left(\mathrm{Al}_{2} \mathrm{O}_{3} / \mathrm{SiO}_{2}\right),\left(\mathrm{K}_{2} \mathrm{O} / \mathrm{Na}_{2} \mathrm{O}\right)$ and $\mathrm{Al}_{2} \mathrm{O}_{3} /\left(\mathrm{CaO}+\mathrm{Na}_{2} \mathrm{O}\right)$ are used to discriminate the plate tectonic setting of sedimentary basins. Bhatia (1983) indicates that the ratio $\mathrm{Al}_{2} \mathrm{O}_{3} /$ 


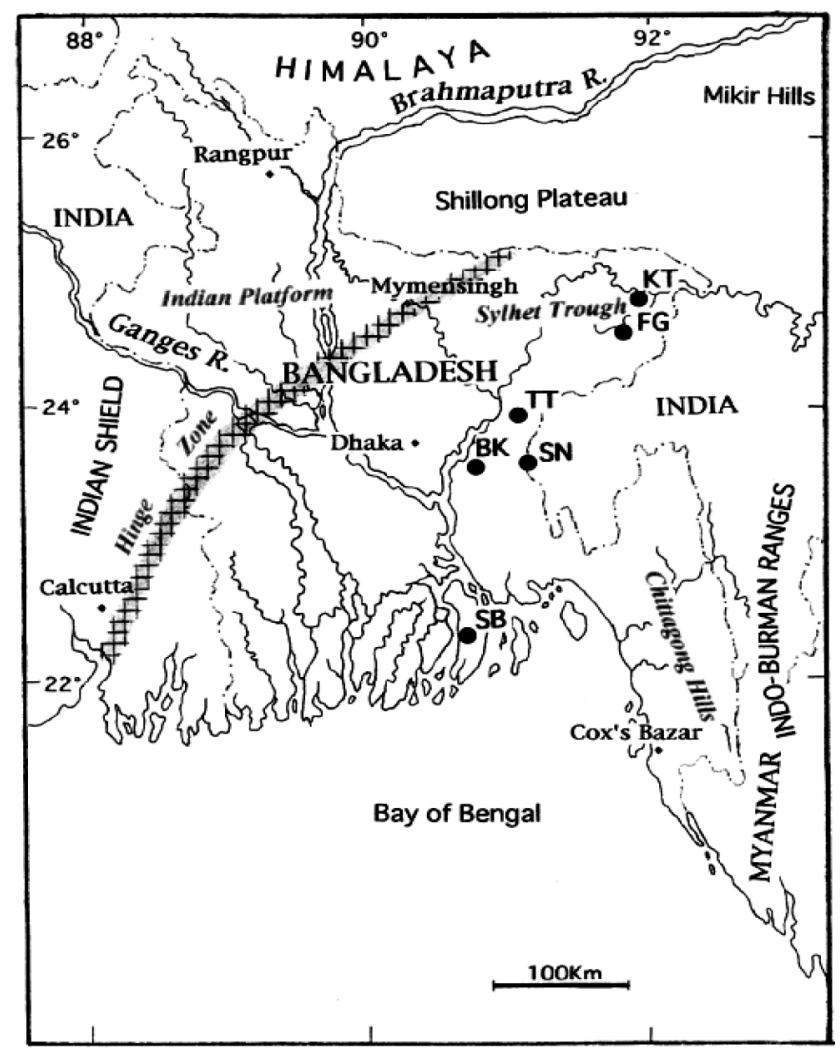

Fig. 1. Major tectonic elements of the Bengal Basin (after Uddin and Lundberg, 1999). The map also shows the locations of petroleum exploration wells: Bakhrabad-10 (BK), Fenchuganj-2 (FG), Kailas Tila-2 (KT), Titas-11 (TT), Shalda Nadi-1 (SN) and Shahbazpur-1 (SB), from which the cores of Miocene Surma Group sandstones were analyzed in this study.

$\mathrm{SiO}_{2}$ gives an indication of the quartz enrichment in sandstones. The ratio $\mathrm{K}_{2} \mathrm{O} / \mathrm{Na}_{2} \mathrm{O}$ is a measure of the potassium feldspar and mica versus plagioclase feldspar content in the rock, and $\mathrm{Al}_{2} \mathrm{O}_{3} /\left(\mathrm{CaO}+\mathrm{Na}_{2} \mathrm{O}\right)$ parameter is a ratio of the most immobile to the most mobile element. Roser and Korsch (1986) used the ratio of $\mathrm{K}_{2} \mathrm{O} /$ $\mathrm{Na}_{2} \mathrm{O}$ and $\mathrm{SiO}_{2}$ content of published data from ancient sedimentary suites to define passive margin (PM), active continental margin (ACM) and oceanic island arc (ARC) settings. For sandstones and argillites of selected New Zealand terranes, Roser and Korsch (1988) used a discriminant function analysis of major elements $\left(\mathrm{TiO}_{2}, \mathrm{Al}_{2} \mathrm{O}_{3}\right.$, total $\mathrm{Fe}_{2} \mathrm{O}_{3}, \mathrm{MgO}, \mathrm{CaO}, \mathrm{Na}_{2} \mathrm{O}$ and $\mathrm{K}_{2} \mathrm{O}$ ) in discriminating four different provenance groups: (1) mafic, (2) intermediate-dominantly andesitic detritus, (3) felsicand plutonic and volcanic detritus and (4) recycledmature polycyclic quartzose detritus.

The elements La, Ce, Nd, Y, Th, Zr, Hf, Nb, Ti and Sc are most suited for provenance and tectonic setting determinations because of their relatively low mobility during sedimentary processes and their low residence time in sea water (Holland, 1978). These elements are transported quantitatively into clastic sedimentary rocks during weathering and transportation, reflecting the signature of the parent materials (Bhatia and Crook, 1986).

The Bengal Basin contains dominantly shallowmarine to continental clastic sediments and minor carbonates, reaching a thickness of about $18 \mathrm{~km}$ of mainly Tertiary to Recent sediments. The collision history of the Himalayan mountain chain, developed along a region once occupied by the west-northwest-south-southeast Tethys ocean, is likely recorded in sediments deposited in subsiding foreland basins, including the Bengal Basin. Sedimentary rocks are principal sources of information that may preserve detritus from orogenic settings. The composition of such clastic materials provides the only available clue to the composition of long-eroded source rocks, and thus is an invaluable tool in sediment source evolution.

The main purpose of this study is to evaluate the major and trace element geochemistry of the Miocene sandstones of the Surma Group from six petroleum exploration wells in the Bengal Basin in order to infer their provenance, tectonic setting and weathering signatures.

\section{Geological SetTing}

The Bengal Basin lies on the north-eastern part of the Indian subcontinent, between the Indian Shield to the west and north, and the Indo-Burman Ranges to the east, and occupies most of Bangladesh, parts of West Bengal and Tripura states of India and the Bay of Bengal (Alam et al., 2003).

The Bengal Basin is well known for the development of a thick $( \pm 22 \mathrm{~km})$ Early Cretaceous-Holocene sedimentary succession (Curray, 1991; Curray and Munasinghe, 1991). The Cretaceous to Holocene Bengal Basin forms a "remnant ocean basin" (Mitchell and Reading, 1986) at the juncture of the Indian plate and the Burma platelet (Curray et al., 1982).

The basin had its origin during the collision of India with Eurasia and Burma, building the extensive Himalayan and Indo-Burman Ranges and thereby loading the lithosphere to form flanking sedimentary basins (Uddin and Lundberg, 1998). Basin development began in the Early Cretaceous when the Indian plate drifted away from Antarctica. Since the Cretaceous Period, sedimentation in the Bengal Basin has been controlled by the movement of the Indian plate with the Burmese and Tibetan plates, and by the uplift and erosion of the Himalayas and Indo-Burmese mountain ranges (Alam, 1989). The onshore part of the Bengal Basin has been divided into platform or shelf, slope or "hinge", and basinal facies (Fig. 1) (Evans, 1964; Salt et al., 1986). Deposition in the Ben- 


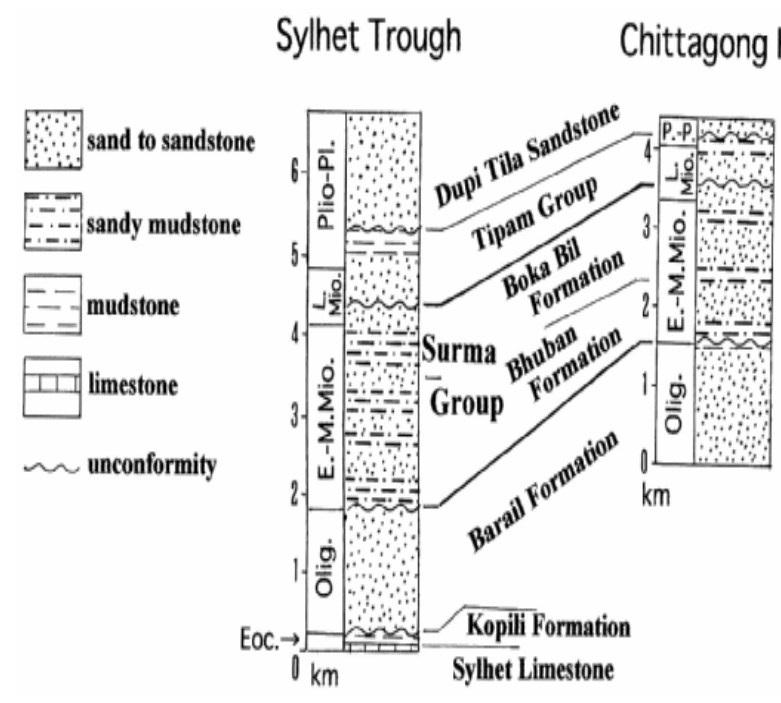

Fig. 2. Stratigraphic framework of the north-east and southeast Bengal Basin (after Uddin and Lundberg, 1999).

gal basin can be explained by two phases of sedimentation (Alam, 1989). The first major phase of sedimentation started with the Cretaceous marine transgression and continued up into the Oligocene period. The collision of the Indian plate with the Tibetan plate and the Burmese plate in the Miocene resulted in a rapid switch in sedimentation pattern in the Bengal Basin. With the Miocene collision of the Indian plate, there was a change from flysch to molasse sedimentation (Alam, 1989).

The Bengal Basin of Bangladesh contains one of the largest delta complexes in the world, covering an area of more than $200,000 \mathrm{~km}^{2}$. It is filled mainly by orogenic sediments derived from the eastern Himalayas to the north and the Indo-Burman ranges to the east (Uddin and Lundberg, 1999) (Fig. 1). These deposits record uplift and exhumation of mountain belts formed by the ongoing India-Eurasia collision (Uddin and Lundberg, 1999). The bulk of the deltaic deposits are Miocene and younger (Fig. 2). Miocene sediments of the Surma Group comprise the early to middle Miocene Bhuban Formation and the middle to late Miocene Boka Bil Formation. These thick accumulations of mudstone and quartzolithic sandstones were derived from neighbouring orogenic belts, and were deposited during repeated transgression and regression. The Neogene Surma Group sediments reach a thickness of $\geq 4 \mathrm{~km}$ in the eastern fold belts and the deeper part of the basin (Uddin and Lundberg, 1999).

\section{METHODS}

Twenty thin sections of the Surma Group sandstones from the central and southeastern Bengal Basin

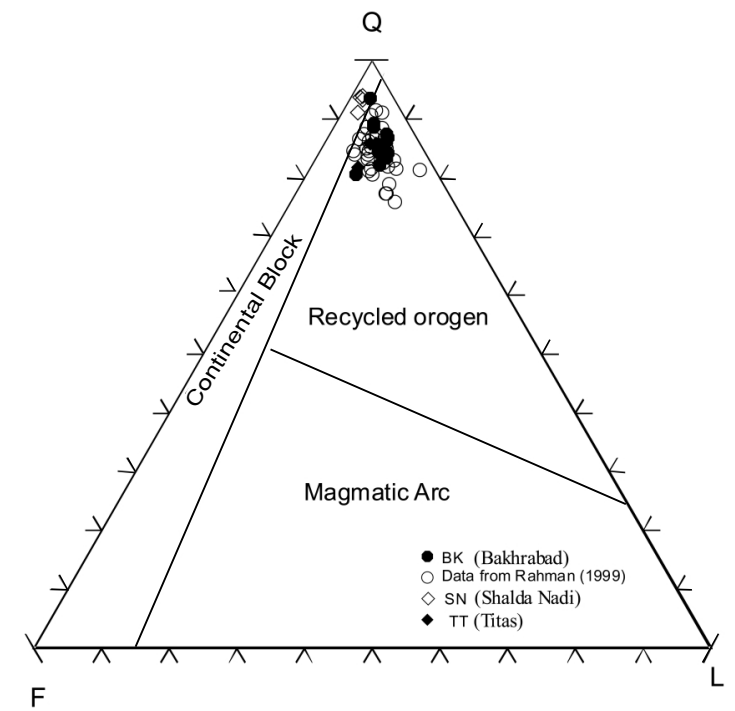

Fig. 3. QFL diagram for the Miocene Surma Group sandstones. Provenance fields after Dickinson (1985).

(Bakhrabad-10, Titas-11 and Shalda Nadi-1) (Fig. 1) were examined by the petrographic microscope, and the modal composition of Surma Group sandstones from the northeastern Bengal Basin (Rahman, 1999) was reviewed. Around 600 counts per thin section were made using the Gazzi-Dickinson point counting method as described by Ingersoll et al. (1984). In the case of coarse mineral aggregates, such as quartz-feldspar aggregates, individual minerals were counted as single grains.

Twenty six sandstone samples of the Miocene Surma Group from six petroleum exploration wells were analyzed for major and trace elements by Activation Laboratories Ltd. (code: 4Lithoresearch), Ontario, Canada. Major elements were analyzed by lithium metaborate/ tetraborate fusion Inductively Coupled Plasma (ICP) and trace and rare earth elements (REE) by Inductively Coupled Plasma Mass Spectrometry (ICP-MS).

\section{RESUlTS}

\section{Petrography}

The Surma Group sandstones are rich in quartz followed by lithic grains, feldspar and mica (predominantly white mica). Quartz is the dominant framework grain, constituting on average $70 \%$ of the rock volume, and occurring as monocrystalline (most abundant) and polycrystalline grains. Feldspars constitute around 7\% of the total framework grains of the sandstones. Both potassium $(\mathrm{K})$ feldspar $(\sim 3 \%)$ and plagioclase $(\mathrm{P})(\sim 3.5 \%)$ are present in almost equal amounts. Next in abundance to quartz, lithic grains make up to $14 \%$ of the total framework grains. Lithic grains are dominated by sedimentary 
Table 1. Chemical composition of the Neogene sandstones of the Surma Group encountered in six petroleum exploration wells in the Bengal Basin (for well locations see Fig. 1). BK-1 = Bakhrabad core 1, FG-5 = Fenchuganj core 5, KT-5 = Kailas Tila core 5, SB-1 = Shahbazpur core 1, SN-2 = Shalda Nadi core 2, TT-1 = Titas core1, BL=Bhuban (lower part), BU=Boka Bil (upper part).

\begin{tabular}{|c|c|c|c|c|c|c|c|}
\hline \multirow{2}{*}{$\begin{array}{l}\text { Sample } \\
\text { Formation }\end{array}$} & \multicolumn{3}{|c|}{ Bakhrabad-10 } & \multicolumn{4}{|c|}{ Fenchuganj-2 } \\
\hline & $\begin{array}{c}\text { BK-1 } \\
\text { BL }\end{array}$ & $\begin{array}{c}\text { BK-2 } \\
\text { BL }\end{array}$ & $\begin{array}{c}\text { BK-3 } \\
\text { BL }\end{array}$ & $\begin{array}{c}\text { FG-5 } \\
\text { BL }\end{array}$ & $\begin{array}{c}\text { FG-10 } \\
\text { BL }\end{array}$ & $\begin{array}{c}\text { FG-13 } \\
\text { BL }\end{array}$ & $\begin{array}{c}\text { FG-15 } \\
\text { BL }\end{array}$ \\
\hline \multicolumn{8}{|l|}{ Major elements } \\
\hline $\mathrm{SiO}_{2}$ & 81.26 & 82.14 & 79.82 & 84.06 & 76.16 & 76.10 & 75.59 \\
\hline $\mathrm{TiO}_{2}$ & 0.433 & 0.504 & 0.498 & 0.408 & 0.631 & 0.617 & 0.413 \\
\hline $\mathrm{Al}_{2} \mathrm{O}_{3}$ & 8.02 & 7.64 & 8.85 & 7.66 & 10.31 & 9.96 & 9.88 \\
\hline $\mathrm{Fe}_{2} \mathrm{O}_{3}$ & 3.39 & 3.24 & 3.55 & 2.06 & 4.15 & 4.01 & 3.71 \\
\hline $\mathrm{MnO}$ & 0.033 & 0.038 & 0.043 & 0.025 & 0.043 & 0.052 & 0.083 \\
\hline $\mathrm{MgO}$ & 0.95 & 0.85 & 1.07 & 0.56 & 1.21 & 1.23 & 1.20 \\
\hline $\mathrm{CaO}$ & 0.45 & 0.51 & 0.67 & 0.55 & 0.60 & 0.94 & 1.97 \\
\hline $\mathrm{Na}_{2} \mathrm{O}$ & 1.24 & 1.24 & 1.41 & 1.39 & 1.88 & 1.93 & 1.97 \\
\hline $\mathrm{K}_{2} \mathrm{O}$ & 1.90 & 1.85 & 2.00 & 1.68 & 2.15 & 2.06 & 1.96 \\
\hline $\mathrm{P}_{2} \mathrm{O}_{5}$ & 0.08 & 0.10 & 0.10 & 0.07 & 0.12 & 0.10 & 0.09 \\
\hline LOI & 1.79 & 1.74 & 2.05 & 1.51 & 2.14 & 2.08 & 3.14 \\
\hline Total & 99.55 & 99.85 & 100.06 & 99.97 & 99.39 & 99.08 & 100.01 \\
\hline $\mathrm{Na}_{2} \mathrm{O} / \mathrm{K}_{2} \mathrm{O}$ & 0.7 & 0.7 & 0.7 & 0.8 & 0.9 & 0.9 & 1.0 \\
\hline $\mathrm{K}_{2} \mathrm{O} / \mathrm{Na}_{2} \mathrm{O}$ & 1.5 & 1.5 & 1.4 & 1.2 & 1.1 & 1.1 & 1.0 \\
\hline $\mathrm{Fe}_{2} \mathrm{O}_{3}+\mathrm{MgO}$ & 4.34 & 4.09 & 4.62 & 2.62 & 5.36 & 5.24 & 4.91 \\
\hline $\mathrm{Al}_{2} \mathrm{O}_{3} / \mathrm{SiO}_{2}$ & 0.10 & 0.09 & 0.11 & 0.09 & 0.14 & 0.13 & 0.13 \\
\hline CIA & 63 & 62 & 62 & 61 & 62 & 59 & 53 \\
\hline \multicolumn{8}{|l|}{ Trace elements } \\
\hline $\mathrm{V}$ & 44 & 43 & 51 & 37 & 60 & 61 & 48 \\
\hline $\mathrm{Cr}$ & 55 & 170 & 70 & 70 & 114 & 94 & 134 \\
\hline $\mathrm{Co}$ & 9 & 8 & 9 & 7 & 12 & 11 & 10 \\
\hline $\mathrm{Ni}$ & 26 & 22 & 26 & 23 & 31 & 36 & 43 \\
\hline $\mathrm{Cu}$ & 18 & & & & 11 & 11 & 13 \\
\hline $\mathrm{Zn}$ & 36 & 35 & 36 & 31 & 55 & 42 & 39 \\
\hline $\mathrm{Ga}$ & 11 & 11 & 12 & 10 & 15 & 15 & 13 \\
\hline $\mathrm{Ge}$ & 1.5 & 1.5 & 1.5 & 1.3 & 1.6 & 1.5 & 1.4 \\
\hline $\mathrm{Rb}$ & 76 & 73 & 80 & 62 & 92 & 88 & 76 \\
\hline $\mathrm{Sr}$ & 76 & 85 & 88 & 104 & 110 & 145 & 139 \\
\hline $\mathrm{Y}$ & 17.5 & 22.1 & 21.4 & 12.9 & 24.3 & 22.6 & 19.8 \\
\hline $\mathrm{Zr}$ & 185 & 256 & 172 & 176 & 229 & 276 & 122 \\
\hline $\mathrm{Nb}$ & 8.0 & 9.2 & 8.7 & 6.9 & 10.7 & 10.6 & 7.4 \\
\hline $\mathrm{Sc}$ & 7 & 7 & 8 & 5 & 9 & 9 & 7 \\
\hline Cs & 2.7 & 2.5 & 2.7 & 1.8 & 3.5 & 3.1 & 2.7 \\
\hline $\mathrm{Ba}$ & 353 & 436 & 324 & 270 & 360 & 396 & 330 \\
\hline $\mathrm{Be}$ & 2 & 2 & 2 & 2 & 2 & 2 & 2 \\
\hline $\mathrm{La}$ & 30.1 & 33.8 & 32.9 & 21.7 & 41.0 & 39.4 & 29.0 \\
\hline $\mathrm{Ce}$ & 62.5 & 69.9 & 69.1 & 44.0 & 84.3 & 80.7 & 61.0 \\
\hline $\operatorname{Pr}$ & 6.48 & 7.26 & 7.30 & 4.45 & 8.84 & 8.31 & 6.44 \\
\hline $\mathrm{Nd}$ & 24.3 & 26.8 & 27.1 & 16.3 & 32.4 & 30.9 & 24.0 \\
\hline $\mathrm{Sm}$ & 4.95 & 5.42 & 5.65 & 3.28 & 6.77 & 6.21 & 5.09 \\
\hline $\mathrm{Eu}$ & 0.998 & 1.06 & 1.14 & 0.628 & 1.31 & 1.16 & 1.13 \\
\hline $\mathrm{Gd}$ & 4.03 & 4.43 & 4.59 & 2.52 & 5.53 & 4.90 & 4.40 \\
\hline $\mathrm{Tb}$ & 0.72 & 0.79 & 0.84 & 0.45 & 1 & 0.87 & 0.8 \\
\hline Dy & 3.79 & 4.3 & 4.43 & 2.53 & 5.18 & 4.63 & 4.21 \\
\hline Ho & 0.69 & 0.81 & 0.81 & 0.48 & 0.94 & 0.86 & 0.78 \\
\hline $\mathrm{Er}$ & 2.05 & 2.53 & 2.39 & 1.49 & 2.88 & 2.55 & 2.31 \\
\hline $\mathrm{Tm}$ & 0.310 & 0.387 & 0.358 & 0.230 & 0.414 & 0.389 & 0.347 \\
\hline $\mathrm{Yb}$ & 1.94 & 2.38 & 2.19 & 1.47 & 2.57 & 2.45 & 2.13 \\
\hline $\mathrm{Lu}$ & 0.277 & 0.351 & 0.311 & 0.223 & 0.379 & 0.365 & 0.306 \\
\hline Hf & 5.2 & 6.7 & 4.7 & 4.9 & 6.6 & 7.4 & 3.5 \\
\hline $\mathrm{Ta}$ & 0.74 & 0.80 & 0.80 & 0.58 & 0.97 & 0.93 & 0.63 \\
\hline W & 1.3 & 1.8 & 1.3 & 1.1 & 2.0 & 1.3 & 2.6 \\
\hline $\mathrm{Tl}$ & 0.67 & 0.59 & 0.66 & 0.59 & 0.83 & 0.74 & 0.66 \\
\hline $\mathrm{Pb}$ & 19 & 20 & 17 & 18 & 21 & 15 & 17 \\
\hline $\mathrm{Bi}$ & 1.4 & 0.8 & 2.4 & 1.2 & 1.0 & 0.9 & 1.1 \\
\hline Th & 9.20 & 11.80 & 10.60 & 10.30 & 13.20 & 13.90 & 8.69 \\
\hline $\mathrm{U}$ & 1.57 & 2.00 & 1.68 & 1.92 & 2.38 & 2.45 & 1.51 \\
\hline$\sum \mathrm{REE}$ & 143 & 160 & 159 & 100 & 194 & 184 & 142 \\
\hline
\end{tabular}


Table 1. (continued)

\begin{tabular}{|c|c|c|c|c|c|c|c|c|c|}
\hline \multirow{2}{*}{$\begin{array}{l}\text { Sample } \\
\text { Formation }\end{array}$} & \multicolumn{4}{|c|}{ Kailas Tila-2 } & \multicolumn{5}{|c|}{ Shahbazpur-1 } \\
\hline & $\begin{array}{c}\text { KT-5 } \\
\text { BU }\end{array}$ & $\begin{array}{c}\text { KT-8 } \\
\text { BL }\end{array}$ & $\begin{array}{c}\text { KT-9 } \\
\text { BL }\end{array}$ & $\begin{array}{c}\mathrm{KT}-10 \\
\mathrm{BL}\end{array}$ & $\begin{array}{c}\text { SB-1 } \\
\text { BU }\end{array}$ & $\begin{array}{c}\text { SB-2 } \\
\text { BU }\end{array}$ & $\begin{array}{c}\text { SB-3 } \\
\text { BU }\end{array}$ & $\begin{array}{c}\text { SB-5 } \\
\text { BL }\end{array}$ & $\begin{array}{c}\text { SB-6 } \\
\text { BL }\end{array}$ \\
\hline \multicolumn{10}{|l|}{ Major elements } \\
\hline $\mathrm{SiO}_{2}$ & 72.92 & 80.50 & 85.23 & 84.31 & 76.71 & 70.06 & 85.09 & 79.38 & 75.34 \\
\hline $\mathrm{TiO}_{2}$ & 0.627 & 0.255 & 0.261 & 0.337 & 0.545 & 0.628 & 0.239 & 0.630 & 0.528 \\
\hline $\mathrm{Al}_{2} \mathrm{O}_{3}$ & 10.35 & 6.66 & 6.59 & 6.51 & 9.26 & 11.16 & 6.89 & 9.03 & 10.07 \\
\hline $\mathrm{Fe}_{2} \mathrm{O}_{3}$ & 4.98 & 2.27 & 2.28 & 2.54 & 3.96 & 5.02 & 2.54 & 3.62 & 4.26 \\
\hline $\mathrm{MnO}$ & 0.085 & 0.163 & 0.023 & 0.037 & 0.039 & 0.066 & 0.020 & 0.040 & 0.046 \\
\hline $\mathrm{MgO}$ & 1.69 & 0.61 & 0.56 & 0.60 & 1.33 & 2.12 & 0.53 & 1.20 & 1.57 \\
\hline $\mathrm{CaO}$ & 1.25 & 2.96 & 0.64 & 0.75 & 0.80 & 2.10 & 0.50 & 0.80 & 0.90 \\
\hline $\mathrm{Na}_{2} \mathrm{O}$ & 1.40 & 1.25 & 1.24 & 1.18 & 1.61 & 1.76 & 1.31 & 1.62 & 1.86 \\
\hline $\mathrm{K}_{2} \mathrm{O}$ & 2.21 & 1.72 & 1.66 & 1.64 & 2.15 & 2.58 & 1.71 & 1.98 & 2.36 \\
\hline $\mathrm{P}_{2} \mathrm{O}_{5}$ & 0.15 & 0.06 & 0.05 & 0.05 & 0.17 & 0.12 & 0.06 & 0.14 & 0.10 \\
\hline LOI & 3.44 & 3.06 & 1.10 & 1.22 & 1.95 & 3.94 & 0.86 & 1.78 & 2.38 \\
\hline Total & 99.10 & 99.51 & 99.63 & 99.17 & 98.52 & 99.55 & 99.75 & 100.22 & 99.41 \\
\hline $\mathrm{Na}_{2} \mathrm{O} / \mathrm{K}_{2} \mathrm{O}$ & 0.6 & 0.7 & 0.7 & 0.7 & 0.7 & 0.7 & 0.8 & 0.8 & 0.8 \\
\hline $\mathrm{K}_{2} \mathrm{O} / \mathrm{Na}_{2} \mathrm{O}$ & 1.6 & 1.4 & 1.3 & 1.4 & 1.3 & 1.5 & 1.3 & 1.2 & 1.3 \\
\hline $\mathrm{Fe}_{2} \mathrm{O}_{3}+\mathrm{MgO}$ & 6.67 & 2.88 & 2.84 & 3.14 & 5.29 & 7.14 & 3.07 & 4.82 & 5.83 \\
\hline $\mathrm{Al}_{2} \mathrm{O}_{3} / \mathrm{SiO}_{2}$ & 0.14 & 0.08 & 0.08 & 0.08 & 0.12 & 0.16 & 0.08 & 0.11 & 0.13 \\
\hline CIA & 61 & 53 & 57 & 57 & 61 & 57 & 59 & 60 & 59 \\
\hline \multicolumn{10}{|l|}{ Trace elements } \\
\hline $\mathrm{V}$ & 66 & 30 & 29 & 33 & 57 & 69 & 30 & 52 & 52 \\
\hline $\mathrm{Cr}$ & 104 & 103 & 104 & 42 & 163 & 61 & 102 & 101 & 86 \\
\hline $\mathrm{Co}$ & 13 & 6 & 6 & 6 & 11 & 12 & 5 & 8 & 9 \\
\hline $\mathrm{Ni}$ & 44 & & & & 35 & 49 & & & \\
\hline $\mathrm{Cu}$ & 20 & & & & 16 & 22 & 12 & & \\
\hline $\mathrm{Zn}$ & 70 & & & & 40 & 66 & & & \\
\hline $\mathrm{Ga}$ & 16 & 9 & 8 & 8 & 13 & 16 & 8 & 11 & 9 \\
\hline $\mathrm{Ge}$ & 1.4 & 1.0 & 1.0 & 1.0 & 1.6 & 1.4 & 1.0 & 1.3 & 1.3 \\
\hline $\mathrm{Rb}$ & 103 & 58 & 53 & 54 & 90 & 126 & 57 & 75 & 76 \\
\hline $\mathrm{Sr}$ & 124 & 166 & 115 & 113 & 121 & 123 & 115 & 115 & 112 \\
\hline $\mathrm{Y}$ & 23.8 & 12.8 & 12.3 & 15.4 & 30.0 & 24.3 & 15.3 & 22.5 & 19.0 \\
\hline $\mathrm{Zr}$ & 269 & 97 & 85 & 124 & 265 & 219 & 100 & 289 & 231 \\
\hline $\mathrm{Nb}$ & 11.1 & 4.8 & 4.6 & 5.3 & 9.5 & 11.5 & 4.2 & 10.0 & 8.3 \\
\hline $\mathrm{Sc}$ & 10 & 5 & 5 & 6 & 9 & 12 & 5 & 9 & 8 \\
\hline Cs & 4.7 & 1.6 & 1.4 & 1.3 & 3.6 & 7.1 & 1.4 & 3.0 & 3.5 \\
\hline $\mathrm{Ba}$ & 382 & 311 & 302 & 373 & 352 & 406 & 295 & 319 & 413 \\
\hline $\mathrm{Be}$ & 2 & 1 & 1 & 1 & 2 & 2 & 1 & 2 & 2 \\
\hline $\mathrm{La}$ & 37.6 & 19.5 & 19.5 & 25.2 & 39.3 & 37.2 & 21.6 & 36.6 & 26.2 \\
\hline $\mathrm{Ce}$ & 77.6 & 39.3 & 40.0 & 52.5 & 81.9 & 75.9 & 45.4 & 76.2 & 53.4 \\
\hline $\operatorname{Pr}$ & 8.17 & 4.10 & 4.20 & 5.50 & 8.70 & 7.86 & 4.80 & 8.09 & 5.52 \\
\hline $\mathrm{Nd}$ & 29.8 & 15.5 & 15.4 & 20.3 & 34.0 & 29.2 & 18.3 & 28.9 & 20.3 \\
\hline $\mathrm{Sm}$ & 6.11 & 3.27 & 3.28 & 4.16 & 7.33 & 6.05 & 3.84 & 5.91 & 4.07 \\
\hline $\mathrm{Eu}$ & 1.20 & 0.749 & 0.778 & 0.850 & 1.50 & 1.19 & 0.838 & 1.08 & 0.785 \\
\hline $\mathrm{Gd}$ & 5.02 & 2.70 & 2.74 & 3.37 & 6.39 & 5.04 & 3.18 & 4.57 & 3.23 \\
\hline $\mathrm{Tb}$ & 0.92 & 0.46 & 0.48 & 0.6 & 1.2 & 0.93 & 0.56 & 0.84 & 0.6 \\
\hline Dy & 4.88 & 2.59 & 2.64 & 3.21 & 6.34 & 4.89 & 2.91 & 4.39 & 3.28 \\
\hline Ho & 0.92 & 0.47 & 0.46 & 0.59 & 1.15 & 0.93 & 0.53 & 0.82 & 0.62 \\
\hline $\mathrm{Er}$ & 2.80 & 1.42 & 1.42 & 1.80 & 3.25 & 2.77 & 1.63 & 2.55 & 1.86 \\
\hline $\mathrm{Tm}$ & 0.431 & 0.204 & 0.210 & 0.272 & 0.467 & 0.423 & 0.233 & 0.385 & 0.292 \\
\hline $\mathrm{Yb}$ & 2.59 & 1.29 & 1.26 & 1.72 & 2.81 & 2.66 & 1.48 & 2.49 & 1.88 \\
\hline $\mathrm{Lu}$ & 0.389 & 0.185 & 0.187 & 0.258 & 0.399 & 0.380 & 0.220 & 0.364 & 0.271 \\
\hline $\mathrm{Hf}$ & 7.7 & 2.7 & 2.4 & 3.5 & 7.3 & 6.4 & 2.9 & 8.2 & 6.2 \\
\hline $\mathrm{Ta}$ & 0.99 & 0.36 & 0.35 & 0.46 & 0.83 & 1.08 & 0.45 & 1.03 & 0.80 \\
\hline W & 1.6 & 0.8 & 1.3 & 0.8 & 1.4 & 2.0 & 0.7 & 1.5 & 1.4 \\
\hline $\mathrm{Tl}$ & 1.12 & 0.45 & 0.52 & 0.50 & 0.81 & 1.25 & 0.55 & 0.67 & 0.05 \\
\hline $\mathrm{Pb}$ & 23 & 26 & 15 & 13 & 21 & 24 & 15 & 17 & 30 \\
\hline $\mathrm{Bi}$ & 2.8 & 0.3 & 0.6 & 0.4 & 1.1 & 3.3 & 0.4 & 1.2 & 2.1 \\
\hline Th & 12.70 & 5.28 & 5.26 & 7.25 & 13.50 & 12.40 & 6.69 & 18.30 & 11.50 \\
\hline $\mathrm{U}$ & 2.54 & 0.98 & 0.98 & 1.19 & 2.17 & 2.35 & 1.28 & 2.87 & 2.02 \\
\hline$\sum \mathrm{REE}$ & 178 & 92 & 93 & 120 & 195 & 175 & 106 & 173 & 122 \\
\hline
\end{tabular}


Table 1. (continued)

\begin{tabular}{|c|c|c|c|c|c|c|c|c|c|c|}
\hline \multirow{2}{*}{$\begin{array}{l}\text { Sample } \\
\text { Formation }\end{array}$} & \multicolumn{5}{|c|}{ Shalda Nadi-1 } & \multicolumn{5}{|c|}{ Titas-11 } \\
\hline & $\begin{array}{c}\mathrm{SN}-2 \\
\mathrm{BU}\end{array}$ & $\begin{array}{c}\text { SN-3 } \\
\text { BU }\end{array}$ & $\begin{array}{c}\text { SN-4 } \\
\text { BU }\end{array}$ & $\begin{array}{c}\text { SN-5 } \\
\text { BL }\end{array}$ & $\begin{array}{c}\text { SN-6 } \\
\text { BL }\end{array}$ & $\begin{array}{c}\text { TT-1 } \\
\text { BL }\end{array}$ & $\begin{array}{c}\text { TT-2 } \\
\text { BL }\end{array}$ & $\begin{array}{c}\text { TT-4 } \\
\text { BL }\end{array}$ & $\begin{array}{c}\text { TT-5 } \\
\text { BL }\end{array}$ & $\begin{array}{c}\text { TT-6 } \\
\text { BL }\end{array}$ \\
\hline \multicolumn{11}{|l|}{ Major elements } \\
\hline $\mathrm{SiO}_{2}$ & 65.34 & 63.50 & 75.02 & 70.72 & 65.71 & 80.79 & 73.56 & 82.58 & 83.25 & 80.75 \\
\hline $\mathrm{TiO}_{2}$ & 0.485 & 0.409 & 0.559 & 0.376 & 0.311 & 0.552 & 0.497 & 0.648 & 0.439 & 0.409 \\
\hline $\mathrm{Al}_{2} \mathrm{O}_{3}$ & 6.41 & 7.21 & 10.56 & 6.57 & 6.12 & 8.03 & 10.97 & 7.63 & 7.71 & 8.28 \\
\hline $\mathrm{Fe}_{2} \mathrm{O}_{3}$ & 2.51 & 2.86 & 5.10 & 3.03 & 2.41 & 3.92 & 5.67 & 3.17 & 2.81 & 3.64 \\
\hline $\mathrm{MnO}$ & 0.808 & 0.188 & 0.055 & 0.242 & 0.671 & 0.075 & 0.056 & 0.053 & 0.037 & 0.073 \\
\hline $\mathrm{MgO}$ & 0.64 & 1.00 & 1.48 & 0.78 & 0.62 & 0.89 & 1.73 & 0.66 & 0.70 & 0.95 \\
\hline $\mathrm{CaO}$ & 10.70 & 11.43 & 0.64 & 8.01 & 11.41 & 0.62 & 0.53 & 0.55 & 0.50 & 0.62 \\
\hline $\mathrm{Na}_{2} \mathrm{O}$ & 1.13 & 1.18 & 1.53 & 1.16 & 1.14 & 1.39 & 1.42 & 1.32 & 1.37 & 1.36 \\
\hline $\mathrm{K}_{2} \mathrm{O}$ & 1.39 & 1.69 & 2.33 & 1.44 & 1.40 & 1.72 & 2.67 & 1.57 & 1.75 & 1.94 \\
\hline $\mathrm{P}_{2} \mathrm{O}_{5}$ & 0.09 & 0.09 & 0.12 & 0.07 & 0.07 & 0.08 & 0.08 & 0.07 & 0.07 & 0.10 \\
\hline LOI & 9.89 & 10.57 & 2.66 & 7.52 & 10.04 & 1.48 & 2.73 & 1.33 & 1.36 & 1.82 \\
\hline Total & 99.39 & 100.13 & 100.05 & 99.92 & 99.90 & 99.55 & 99.91 & 99.58 & 100.00 & 99.94 \\
\hline $\mathrm{Na}_{2} \mathrm{O} / \mathrm{K}_{2} \mathrm{O}$ & 0.8 & 0.7 & 0.7 & 0.8 & 0.8 & 0.8 & 0.5 & 0.8 & 0.8 & 0.7 \\
\hline $\mathrm{K}_{2} \mathrm{O} / \mathrm{Na}_{2} \mathrm{O}$ & 1.2 & 1.4 & 1.5 & 1.2 & 1.2 & 1.2 & 1.9 & 1.2 & 1.3 & 1.4 \\
\hline $\mathrm{Fe}_{2} \mathrm{O}_{3}+\mathrm{MgO}$ & 3.15 & 3.86 & 6.58 & 3.81 & 3.03 & 4.81 & 7.40 & 3.83 & 3.51 & 4.59 \\
\hline $\mathrm{Al}_{2} \mathrm{O}_{3} / \mathrm{SiO}_{2}$ & 0.10 & 0.11 & 0.14 & 0.09 & 0.09 & 0.10 & 0.15 & 0.09 & 0.09 & 0.10 \\
\hline CIA & 55 & 56 & 64 & 55 & 54 & 61 & 65 & 62 & 61 & 61 \\
\hline \multicolumn{11}{|l|}{ Trace elements } \\
\hline $\mathrm{V}$ & 32 & 36 & 63 & 33 & 28 & 45 & 60 & 50 & 37 & 42 \\
\hline $\mathrm{Cr}$ & 67 & 97 & 69 & 120 & 35 & 142 & 53 & 138 & 53 & 87 \\
\hline $\mathrm{Co}$ & 4 & 5 & 14 & 5 & 4 & 7 & 21 & 6 & 5 & 8 \\
\hline $\mathrm{Ni}$ & 24 & & 69 & 21 & & 20 & 52 & & & 24 \\
\hline $\mathrm{Cu}$ & & & 12 & & & & 11 & & & \\
\hline $\mathrm{Zn}$ & & & 45 & & & & 51 & & 34 & \\
\hline $\mathrm{Ga}$ & 8 & 9 & 14 & 8 & 7 & 10 & 15 & 9 & 9 & 10 \\
\hline $\mathrm{Ge}$ & 1.0 & 1.1 & 1.4 & 1.2 & 0.9 & 1.3 & 1.4 & 1.2 & 1.2 & 1.1 \\
\hline $\mathrm{Rb}$ & 50 & 68 & 100 & 54 & 49 & 63 & 116 & 55 & 58 & 71 \\
\hline $\mathrm{Sr}$ & 315 & 311 & 98 & 392 & 384 & 110 & 96 & 101 & 102 & 100 \\
\hline $\mathrm{Y}$ & 23.7 & 21.9 & 22.8 & 19.0 & 14.0 & 16.6 & 16.7 & 24.0 & 15.9 & 16.1 \\
\hline $\mathrm{Zr}$ & 313 & 195 & 176 & 157 & 108 & 234 & 127 & 230 & 188 & 141 \\
\hline $\mathrm{Nb}$ & 7.7 & 7.8 & 10.0 & 6.8 & 5.4 & 9.1 & 9.4 & 10.3 & 7.2 & 6.8 \\
\hline $\mathrm{Sc}$ & 6 & 6 & 10 & 5 & 5 & 7 & 10 & 8 & 7 & 8 \\
\hline Cs & 1.7 & 2.8 & 4.9 & 1.8 & 1.5 & 2.1 & 6.1 & 1.6 & 1.8 & 2.6 \\
\hline $\mathrm{Ba}$ & 238 & 286 & 365 & 235 & 233 & 307 & 395 & 312 & 496 & 336 \\
\hline $\mathrm{Be}$ & 2 & 2 & 2 & 2 & 2 & 2 & 2 & 2 & 2 & 2 \\
\hline $\mathrm{La}$ & 30.6 & 25.4 & 30.3 & 22.0 & 15.1 & 26.9 & 22.9 & 40.1 & 29.7 & 23.1 \\
\hline $\mathrm{Ce}$ & 61.9 & 51.8 & 62.6 & 45.7 & 29.8 & 56.8 & 48.3 & 84.6 & 63.2 & 48.2 \\
\hline $\operatorname{Pr}$ & 6.46 & 5.50 & 6.68 & 4.89 & 3.04 & 5.77 & 5.12 & 8.77 & 6.56 & 5.06 \\
\hline $\mathrm{Nd}$ & 23.5 & 20.8 & 25.5 & 18.1 & 11.2 & 20.7 & 19.8 & 31.8 & 23.8 & 18.7 \\
\hline $\mathrm{Sm}$ & 4.82 & 4.37 & 5.37 & 3.87 & 2.28 & 4.21 & 4.26 & 6.35 & 4.67 & 3.72 \\
\hline $\mathrm{Eu}$ & 0.900 & 0.923 & 1.14 & 0.761 & 0.515 & 0.797 & 0.973 & 1.17 & 0.912 & 0.796 \\
\hline $\mathrm{Gd}$ & 3.98 & 3.82 & 4.49 & 3.26 & 2.08 & 3.24 & 3.63 & 4.83 & 3.60 & 3.08 \\
\hline $\mathrm{Tb}$ & 0.72 & 0.69 & 0.85 & 0.62 & 0.39 & 0.59 & 0.66 & 0.85 & 0.61 & 0.59 \\
\hline Dy & 4.12 & 3.95 & 4.43 & 3.41 & 2.43 & 3.26 & 3.45 & 4.54 & 3.19 & 3.15 \\
\hline Ho & 0.82 & 0.72 & 0.81 & 0.64 & 0.49 & 0.64 & 0.64 & 0.85 & 0.57 & 0.6 \\
\hline $\mathrm{Er}$ & 2.50 & 2.21 & 2.45 & 1.99 & 1.50 & 1.99 & 1.96 & 2.65 & 1.77 & 1.82 \\
\hline $\mathrm{Tm}$ & 0.392 & 0.319 & 0.358 & 0.307 & 0.235 & 0.316 & 0.280 & 0.404 & 0.272 & 0.277 \\
\hline $\mathrm{Yb}$ & 2.43 & 1.99 & 2.17 & 1.91 & 1.44 & 2.05 & 1.71 & 2.57 & 1.71 & 1.74 \\
\hline $\mathrm{Lu}$ & 0.354 & 0.290 & 0.327 & 0.284 & 0.205 & 0.314 & 0.256 & 0.378 & 0.246 & 0.262 \\
\hline Hf & 8.9 & 5.2 & 5.0 & 4.4 & 3.1 & 6.5 & 3.7 & 6.3 & 5.1 & 4.1 \\
\hline $\mathrm{Ta}$ & 0.71 & 0.75 & 0.87 & 0.59 & 0.46 & 0.90 & 0.88 & 0.89 & 0.64 & 0.61 \\
\hline W & 0.9 & 15.8 & 2.3 & 0.8 & 0.8 & 1.3 & 1.8 & 1.3 & 1.0 & 1.1 \\
\hline $\mathrm{Tl}$ & 0.49 & 0.65 & 0.95 & 0.57 & 0.41 & 0.68 & 1.06 & 0.54 & 0.60 & 0.80 \\
\hline $\mathrm{Pb}$ & 15 & 18 & 19 & 22 & 15 & 24 & 27 & 17 & 22 & 30 \\
\hline $\mathrm{Bi}$ & 0.5 & 0.9 & 0.6 & 2.2 & 1.6 & 4.7 & 5.0 & 0.9 & 3.5 & 5.8 \\
\hline $\mathrm{Th}$ & 13.00 & 9.40 & 10.50 & 8.81 & 6.20 & 12.20 & 8.54 & 15.30 & 9.92 & 8.88 \\
\hline $\mathrm{U}$ & 2.20 & 1.63 & 1.85 & 1.39 & 1.16 & 1.94 & 1.35 & 2.21 & 1.58 & 1.43 \\
\hline$\sum \mathrm{REE}$ & 143 & 123 & 147 & 108 & 71 & 128 & 114 & 190 & 141 & 111 \\
\hline
\end{tabular}

Major elements in wt.\%, trace elements in ppm. 

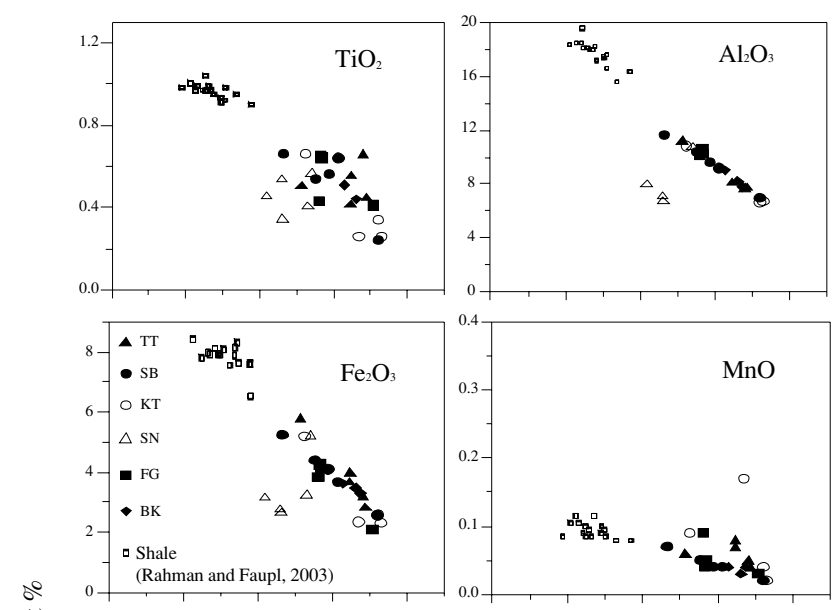

3
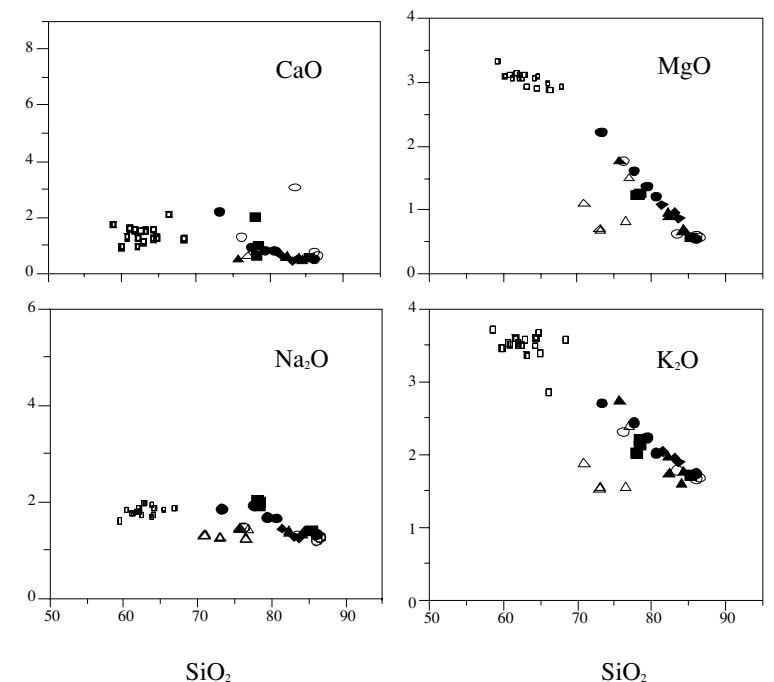

Fig. 4. Harker variation diagrams for major elements in the Surma Group sediments.

(mainly chert, shale) and low-grade metamorphic rocks (mainly fine-grained mica schist, quartz-mica schist and phyllites, partly graphite-bearing), along with a few volcanic rock fragments. Phyllosilicates constitute about $6 \%$ of the framework grains. White mica is generally more abundant $(3.6 \%)$ than biotite $(2.3 \%)$. Chlorite constitutes about $1.5 \%$ of the total framework grains. Calcite and dolomite are found both as detrital grains and as cements. Dolomite is predominant among the carbonate minerals, constituting about $1.1 \%$, whereas calcite constitutes around $0.5 \%$. Iron-bearing calcites are common.

The heavy mineral assemblage is characterized by predominance of garnet followed by apatite, tourmaline, epidote and staurolite.

The Surma Group sandstones have dominantly quartzolithic composition with predominance of quartz. Some feldspathic sandstones and quartz arenites are also recognized. The Surma sandstones contain abundant low-

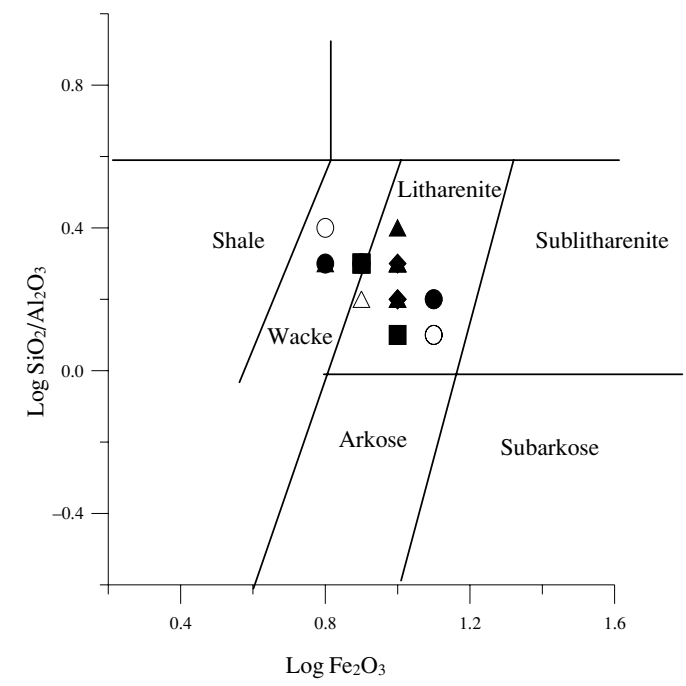

Fig. 5. Chemical classification of the Miocene Surma Group sandstones (after Herron, 1986).

grade metamorphic, and sedimentary lithics, and little feldspar and volcanic detritus, indicating that the sands were derived from a quartzose recycled orogen province (Fig. 3).

\section{Chemical composition}

Averaged major and trace element analyses of the sandstones of the Surma Group from different wells are presented in Table 1.

Major elements The Surma Group sandstones have moderate to high $\mathrm{SiO}_{2}$ contents (64-85\%; on average 77\%), $\mathrm{TiO}_{2}$ concentrations averaging $0.5 \%, \mathrm{Al}_{2} \mathrm{O}_{3}$ contents of about $8.4 \%$, and $\mathrm{Fe}_{2} \mathrm{O}_{3}$ (total $\mathrm{Fe}$ as $\mathrm{Fe}_{2} \mathrm{O}_{3}$ ) $+\mathrm{MgO}$ content of around $4.5 \%$. Compared to the average sandstone value proposed by Turekian and Wedepohl (1961), the Surma Group sandstones are depleted in $\mathrm{CaO}(2.36 \%)$ and enriched in $\mathrm{Al}_{2} \mathrm{O}_{3}, \mathrm{Fe}_{2} \mathrm{O}_{3}$ and $\mathrm{Na}_{2} \mathrm{O}$. The samples show no marked differences in major chemical composition. The only exception is in their $\mathrm{CaO}$ content. Surma Group sandstones in the Shaldha Nadi well (SN in Fig. 1) are rich in $\mathrm{CaO}$ content which is agreement with petrographic observations. Most of the Shaldha Nadi sandstones are cemented with calcite. The linear relationship of $\mathrm{TiO}_{2}, \mathrm{Al}_{2} \mathrm{O}_{3}, \mathrm{Fe}_{2} \mathrm{O}_{3}, \mathrm{MgO}$ and $\mathrm{K}_{2} \mathrm{O}$ with $\mathrm{SiO}_{2}$ in the Surma Group sediments is conspicuous in the Harker variation diagrams, both lithologies (sandstones and shales) show similar linear trend (Fig. 4). The negative correlation of $\mathrm{SiO}_{2}$ with most major elements is due to most of the silica being sequestered in quartz, as indicated by Osman (1996). In the present samples, $\mathrm{TiO}_{2}$ concentrations increase with $\mathrm{Al}_{2} \mathrm{O}_{3}$, suggesting that $\mathrm{TiO}_{2}$ is probably associated with phyllosilicates especially with illite (Dabard, 1990); $\mathrm{Fe}_{2} \mathrm{O}_{3}+\mathrm{MgO}$ are also well corre- 


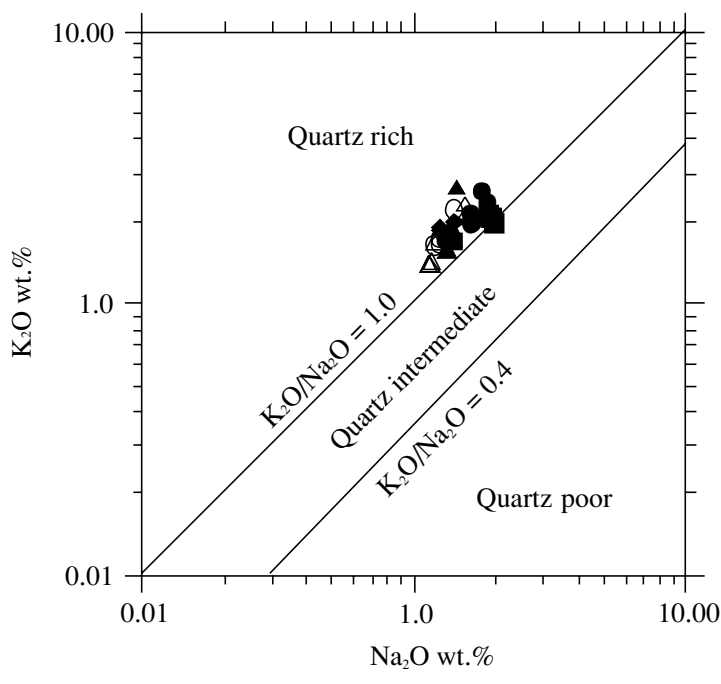

Fig. 6. Analysis of sandstones according to the richness of quartz of the Miocene Surma Group (after Crook, 1974).

lated with $\mathrm{Al}_{2} \mathrm{O}_{3}$. The latter correlation implies that these oxides are associated with phyllosilicates, particularly in matrix chlorites (Dabard, 1990).

According to the diagram of Herron (1986) the sandstones are classified mainly as litharenites (Fig. 5). On the $\mathrm{Na}_{2} \mathrm{O}-\mathrm{K}_{2} \mathrm{O}$ diagram (Fig. 6), the Surma Group sandstones data plot in the quartz-rich field.

Trace elements Trace element concentrations of the Surma Group sandstones are in concurrence with average upper continental crust (UCC) with the exception of $\mathrm{Ni}, \mathrm{Cr}, \mathrm{Sr}$ and $\mathrm{Nb}$ content (Fig. 7). In the present samples, $\mathrm{Ni}, \mathrm{Cr}$ are enriched and $\mathrm{Sr}, \mathrm{Nb}$ are depleted. $\mathrm{The} \mathrm{Cr}$ enrichment (35-170 ppm) is related to the presence of chrome spinel which is reflected in the petrographic observation. The Sr content (76-392 ppm) of sedimentary rocks is variable because of the many influences on $\mathrm{Sr}$ in low temperature depositional environments (Fairbridge, 1972), e.g., the distribution of Sr can be affected by the presence of $\mathrm{Ca}$, additional $\mathrm{Sr}$ can be incorporated in diagenetic carbonate, and fractionation of $\mathrm{Sr}$ can result from the weathering of feldspars, particularly plagioclase. Clear positive correlations between $\mathrm{K}$ contents and the abundances of $\mathrm{Al}, \mathrm{Cs}, \mathrm{Ba}$, total $\mathrm{REE}$, Th and $\mathrm{U}$ suggest that the concentrations of these trace elements are controlled by clay minerals and mica (after McLennan et al., 1983). A significant correlation $\left(r^{2}=0.63\right)$ between $\mathrm{Ba}$ and $\mathrm{K}_{2} \mathrm{O}$ suggests that $\mathrm{Ba}$ is mainly associated with a feldspar component. High field strength elements (e.g., Zr, $\mathrm{Nb}, \mathrm{Hf}, \mathrm{Y}$ ) generally show consistent inter-relationships, as do the ferromagnesian trace elements $(\mathrm{Cr}, \mathrm{Ni}, \mathrm{V}, \mathrm{Co}$, and $\mathrm{Sc}$ ). These trace element relationships illustrate the chemical coherence and uniformity of the sediments. Cobalt and Sc abundances show significant positive cor-

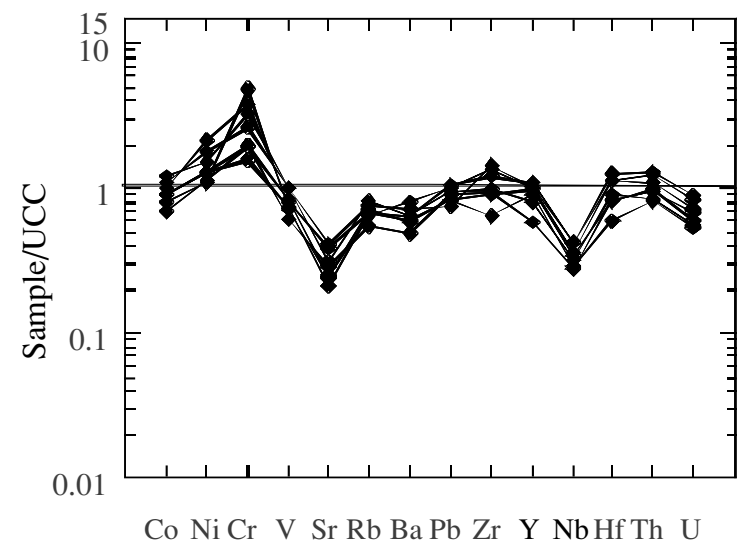

Fig. 7. Multi-element normalized diagram for the Surma Group sandstones, normalized against upper continental crust (Taylor and McLennan, 1985).

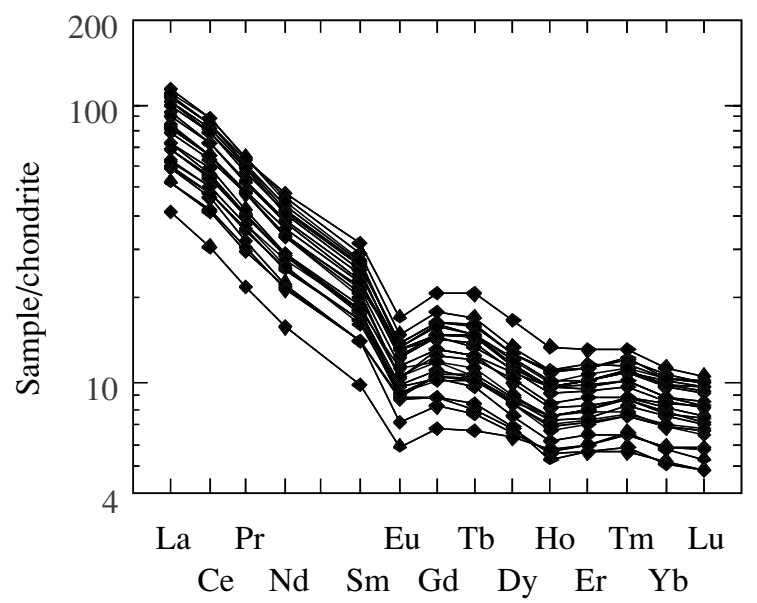

Fig. 8. Chondrite-normalized rare earth element plots for the Surma Group sandstones. Chondrite values from Taylor and McLennan (1985).

relation with $\mathrm{Ni}, \mathrm{V}$ and $\mathrm{Al}_{2} \mathrm{O}_{3}$ inferring that $\mathrm{Co}$ and $\mathrm{Sc}$ are partly controlled by chlorite and other accessory nonaluminous silicate minerals.

Rare Earth Elements REE concentrations of the Surma Group sandstones are shown as chondrite-normalized patterns in Fig. 8. The sandstones have (REE contents ranging between 71-195 with an average of 139, comparable to average UCC (143, Taylor and McLennan 1985). The sandstones show slight LREE-enriched and relatively flat HREE patterns with negative Eu anomalies.

\section{DISCUSSION}

Provenance and tectonic setting

Several classifications have been proposed to discrimi- 
nate from various origins and tectonic settings (Maynard et al., 1982; Bhatia, 1983; Bhatia and Crook, 1986; Roser and Korsch, 1986, 1988). To infer provenance, unstandardised discriminant function scores of the samples (F1 and F2) for major elements were plotted following the boundaries between fields (P1-P4), as proposed by Roser and Korsch (1988) (Fig. 9). The Surma Group sandstones and shales fall within the P4 field, which represents recycled mature polycyclic quartzose detritus. Recycled sources represent quartzose sediments of mature continental provenance, and the derivation of the sediments could be from a highly weathered granitegneiss terrain and/or from a pre-existing sedimentary terrane as in the case of the Greenland Group of New

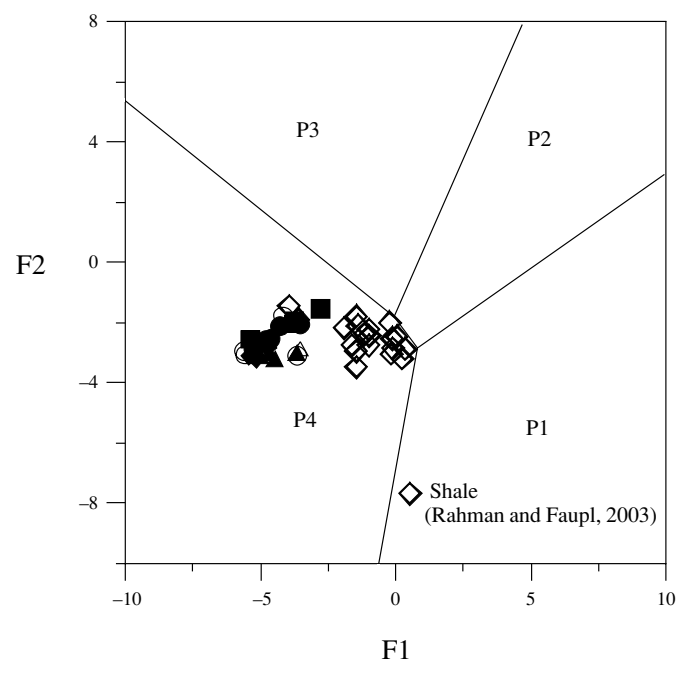

Fig. 9. Plot of discriminant functions F1 and F2 for the sandstones from the Miocene Surma Group of the Bengal Basin. Provenance fields are after Roser and Korsch (1988). PI = mafic and lesser intermediate igneous provenance, $P 2=i n$ termediate igneous provenance; $P 3=$ felsic igneous provenance and $\mathrm{P} 4$ = recycled-mature polycyclic quartzose detritus.
Zealand (Roser and Korsch, 1988). The recycled nature of the Surma Group is also reflected in the sandstone modal compositions (Fig. 3). The Surma Group sandstones in the Bengal Basin have dominantly quartzolithic composition with predominance of quartz, abundant low-grade metamorphic and sedimentary lithics, less feldspar and little volcanic detritus. The ${ }^{40} \mathrm{Ar} /{ }^{39} \mathrm{Ar}$ results for the Surma Group sandstones point to heterogeneous ages of the source region, which seems to be typical of the early stages of a rapidly uplifting collision orogen (Rahman and Faupl, 2003a). The narrow age range (cluster between 25 and $36 \mathrm{Ma}$ ) of the low temperature steps can be correlated with early cooling ages known from the High Himalayan crystalline basement (Rahman and Faupl, 2003a). The concentrations of Rb (mean $\sim 74$ ppm), Sr $(\sim 148), \mathrm{Sm}(\sim 5.0)$ and $\mathrm{Nd}(\sim 23)$ in the Surma Group sandstones are in accordance with those of High Himalaya sedimentary series (France-Lanord et al., 1993).

The high-field-strength elements (HFSE) such as Zr, $\mathrm{Nb}, \mathrm{Hf}, \mathrm{Y}, \mathrm{Th}$ are preferentially partitioned into melts during crystallization (Feng and Kerrich, 1990), and as a result these elements are enriched in felsic rather than mafic sources. These elements are thought to reflect provenance compositions as a consequence of their generally immobile behavior (Taylor and McLennan, 1985). The REE and Sc also give indication of source compositions because of their relatively low mobility during sedimentation and their low residence times in sea water (Bhatia and Crook, 1986). REE and Th abundances are higher in felsic than in mafic igneous source rocks and in their weathered products, whereas $\mathrm{Co}, \mathrm{Sc}$, and $\mathrm{Cr}$ are more concentrated in mafic than in felsic igneous rocks and in their weathered products. Mafic and felsic source rocks differ significantly in the ratios such of $\mathrm{Eu} / \mathrm{Eu}^{*}$, (La/ $\mathrm{Lu}) \mathrm{cn}, \mathrm{La} / \mathrm{Sc}, \mathrm{Th} / \mathrm{Sc}, \mathrm{La} / \mathrm{Co}, \mathrm{Th} / \mathrm{Co}$, and $\mathrm{Cr} / \mathrm{Th}$ and hence provide useful information about the provenance of sedimentary rocks (e.g., Cullers et al., 1988; Cullers, 1994, 2000; Cullers and Podkovyrov, 2000). In this study, Eu/ $\mathrm{Eu}^{*},(\mathrm{La} / \mathrm{Lu}) \mathrm{cn}, \mathrm{La} / \mathrm{Sc}, \mathrm{Th} / \mathrm{Sc}, \mathrm{La} / \mathrm{Co}, \mathrm{Th} / \mathrm{Co}$, and $\mathrm{Cr} / \mathrm{Th}$

Table 2. Range of elemental ratios of Miocene Surma Group sandstones in this study compared to elemental ratios in sediments derived from felsic rocks, mafic rocks, and in the upper continental crust

\begin{tabular}{lcccc}
\hline Elemental ratio & $\begin{array}{c}\text { Surma Group sandstones } \\
(n=26)\end{array}$ & $\begin{array}{c}\text { Ranges in sediments } \\
\text { from felsic sources }{ }^{1)}\end{array}$ & $\begin{array}{c}\text { Ranges in sediments } \\
\text { from mafic sources }{ }^{1)}\end{array}$ & $\begin{array}{c}\left.\text { Upper Continental Crust }^{2}\right) \\
\text { Eu/Eu* }\end{array}$ \\
$(\mathrm{La} / \mathrm{Lu}) \mathrm{cn}$ & $0.63-0.77$ & $0.40-0.94$ & $0.71-0.95$ & 0.63 \\
$\mathrm{La} / \mathrm{Sc}$ & $7.65-11.28$ & $3.00-27.0$ & $1.10-7.00$ & 9.73 \\
$\mathrm{Th} / \mathrm{Sc}$ & $2.89-5.01$ & $2.50-16.3$ & $0.43-0.86$ & 2.21 \\
$\mathrm{La} / \mathrm{Co}$ & $0.85-2.17$ & $0.84-20.5$ & $0.05-0.22$ & 0.79 \\
$\mathrm{Th} / \mathrm{Co}$ & $1.09-7.65$ & $1.80-13.8$ & $0.14-0.38$ & 1.76 \\
$\mathrm{Cr} / \mathrm{Th}$ & $0.41-3.25$ & $0.04-3.25$ & $0.04-1.40$ & 0.63 \\
\hline
\end{tabular}

${ }^{1)}$ Cullers et al. (1988); Cullers (1994, 2000); Cullers and Podkovyrov (2000).

${ }^{2)}$ McLennan (2001); Taylor and McLennan (1985). 


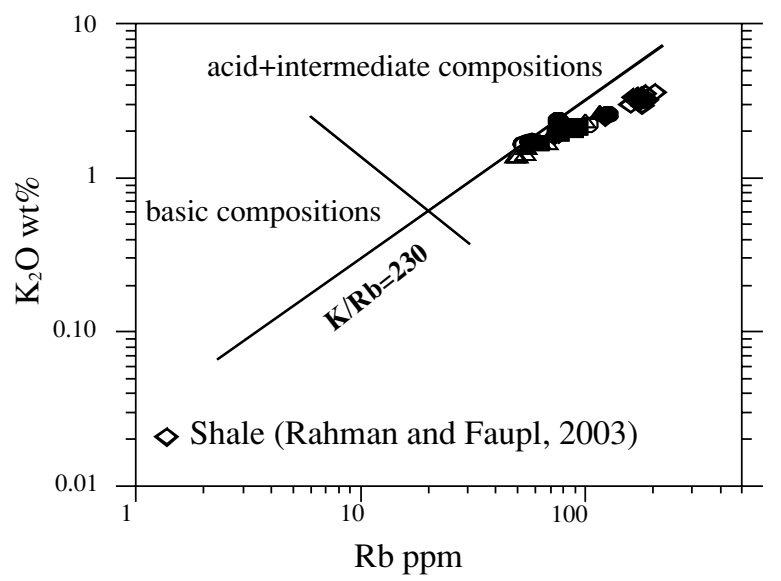

Fig. 10. Distribution of $K$ and $R b$ in the Miocene Surma Group sediments relative to a $K / R b$ ratio of 230 (=main trend of Shaw, 1968).

values of the Miocene Surma Group sandstones are more similar to values for sediments derived from felsic source rocks than to those for mafic source rocks (Table 2), suggesting derivation from felsic source rocks. The higher LREE/HREE ratios and negative Eu anomalies of the Surma Group sandstones also bears the characteristics of felsic source rocks (after Taylor and McLennan 1985; Wronkiewicz and Condie 1989). The Surma Group sediments have uniform $\mathrm{K} / \mathrm{Rb}$ ratios that lie close to a typical differentiated magmatic suite or main trend with a ratio of 230 (Fig. 10) (Shaw, 1968). This characteristic reveals the chemically coherent nature of the sediments and derivation from rocks of acidic and intermediate compositions.

The ferromagnesian trace elements $\mathrm{Cr}, \mathrm{Ni}, \mathrm{Co}$, and $\mathrm{V}$ show generally similar behavior in magmatic processes, but they may be fractionated during weathering (Feng and Kerrich, 1990). In the studied samples, $\mathrm{Cr}$ and Ni are enriched with respect to the average composition of the Upper Continental Crust (UCC). This enrichment in $\mathrm{Cr}$ and Ni may suggest some basic input from the source terrane.

The elevated values of $\mathrm{Cr}(>150 \mathrm{ppm})$ and $\mathrm{Ni}(>100$ $\mathrm{ppm}$ ) and a ratio of $\mathrm{Cr} / \mathrm{Ni}$ between 1.3-1.5 are diagnostic of ultramafic rocks in the source region (Garver et al., 1996). In comparison, $\mathrm{Cr}$ concentrations ranges from 35 to $170 \mathrm{ppm}$ (average $94 \mathrm{ppm}$ ) and $\mathrm{Ni}$ concentrations ranges from 20 to $69 \mathrm{ppm}$ (average $34 \mathrm{ppm}$ ). $\mathrm{Cr} / \mathrm{Ni}$ ratios vary from 1.0 to 7.7 , but are mostly less than 2.0. This comparison implies that the existence of widespread mafic/ultramafic rocks in the source region was most unlikely. The occurrence of only rare detrital chrome spinel $(\sim 0.3 \%)$ within the sandstones of the Surma Group, as revealed from heavy mineral data, is in good agreement with this finding.

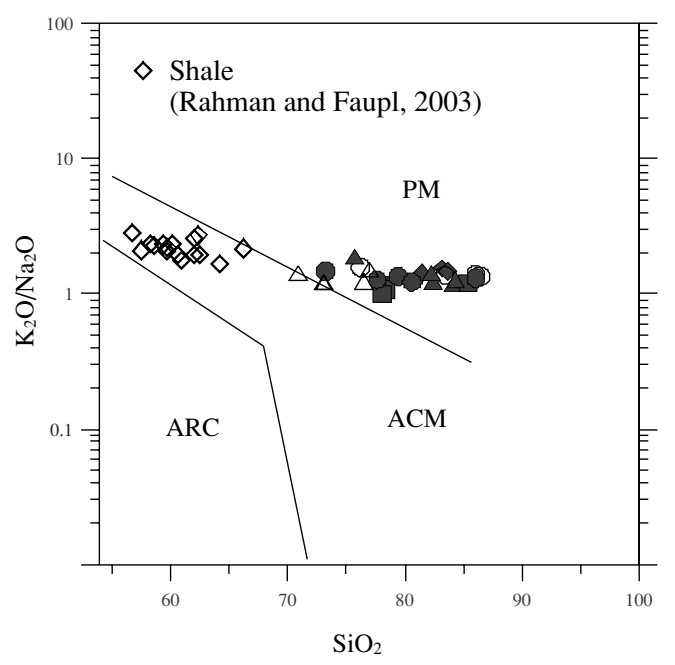

Fig. 11. Tectonic discrimination diagram for Surma Group sediments. Boundaries are after Roser and Korsch, 1986. PM = passive margin, ACM = active continental margin, $A R C=$ oceanic island arc margin.

Roser and Korsch (1986) established a discrimination diagram using $\log \left(\mathrm{K}_{2} \mathrm{O} / \mathrm{Na}_{2} \mathrm{O}\right)$ versus $\mathrm{SiO}_{2}$ (Fig. 11) to determine the tectonic setting of terrigenous sedimentary rocks. $\mathrm{SiO}_{2}$ and $\mathrm{K}_{2} \mathrm{O} / \mathrm{Na}_{2} \mathrm{O}$ increase from volcanic-arc to active continental margin to passive margin settings. The Surma Group sandstones plot in the passive margin field, whereas the shale samples fall in the active continental margin field (Fig. 11). Discrimination of tectonic settings on the basis of major element data was also proposed by Bhatia (1983); these include oceanic island arc, continental island arc, active continental margin, and passive margin settings. Most of the Surma Group sandstones fall in the general area of active continental margin and passive margin fields of the $\mathrm{Al}_{2} \mathrm{O}_{3} / \mathrm{SiO}_{2}$ versus $\mathrm{Fe}_{2} \mathrm{O}_{3}+\mathrm{MgO}$; $\mathrm{K} 2 \mathrm{O} / \mathrm{Na}_{2} \mathrm{O}$ versus $\mathrm{Fe}_{2} \mathrm{O}_{3}+\mathrm{MgO} ; \mathrm{Al}_{2} \mathrm{O}_{3} /\left(\mathrm{CaO}+\mathrm{Na}_{2} \mathrm{O}\right)$ versus $\mathrm{Fe}_{2} \mathrm{O}_{3}+\mathrm{MgO}$ (Fig. 12) but there is much scatter on plot the $\mathrm{TiO}_{2}$ versus $\mathrm{Fe}_{2} \mathrm{O}_{3}+\mathrm{MgO}$ plot, with data spreading across three fields (active continental margin, continental island arc, passive margin).

\section{Weathering in the source area}

The Chemical Index of Alteration (CIA) gives an indication of the degree of weathering in the source region (Nesbitt and Young, 1982). CIA records the progressive alteration of plagioclase and potassium feldspars to clay minerals. The CIA index is calculated using molar proportions following the equation CIA $=\left[\mathrm{Al}_{2} \mathrm{O}_{3} /\left(\mathrm{Al}_{2} \mathrm{O}_{3}+\right.\right.$ $\left.\left.\mathrm{CaO} *+\mathrm{Na}_{2} \mathrm{O}+\mathrm{K}_{2} \mathrm{O}\right)\right] * 100$, where $\mathrm{CaO} *$ represents the amount of $\mathrm{CaO}$ incorporated in the silicate phases. Nesbitt and Young (1982) suggested a CIA value of nearly 100 for kaolinite and chlorite, and 70-75 for average shales. High values (i.e., 76-100) indicate intensive chemical 

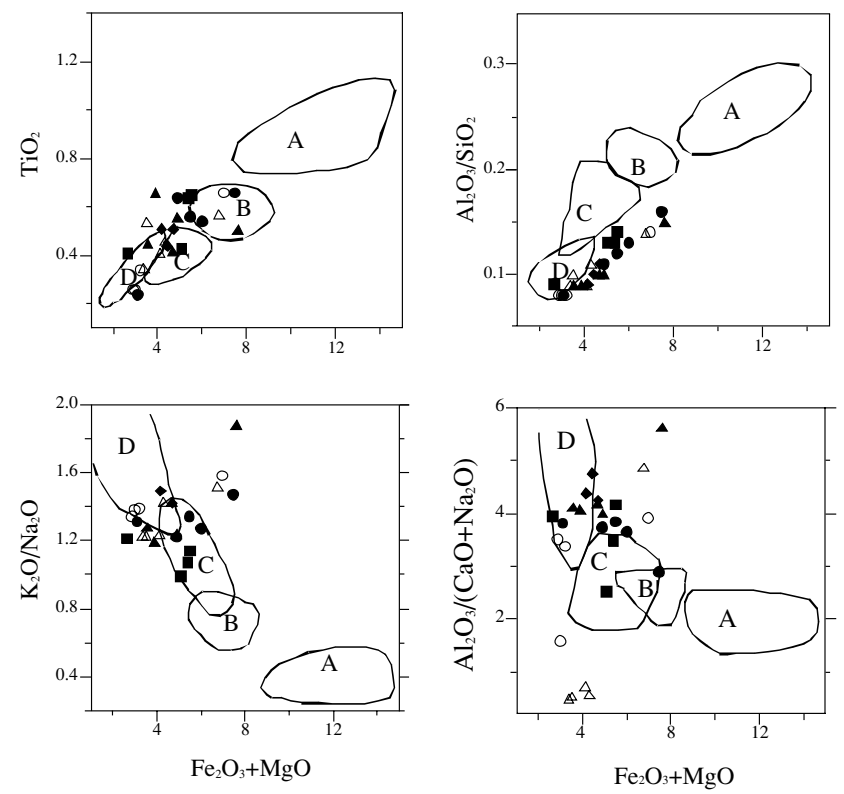

Fig. 12. Tectonic-setting discrimination diagrams for Surma Group sediments. Boundaries are after Bhatia (1983). A =Oceanic island arc margin; $B=$ Continental island arc; $C=$ Active continental margin; $D=$ Passive margin.

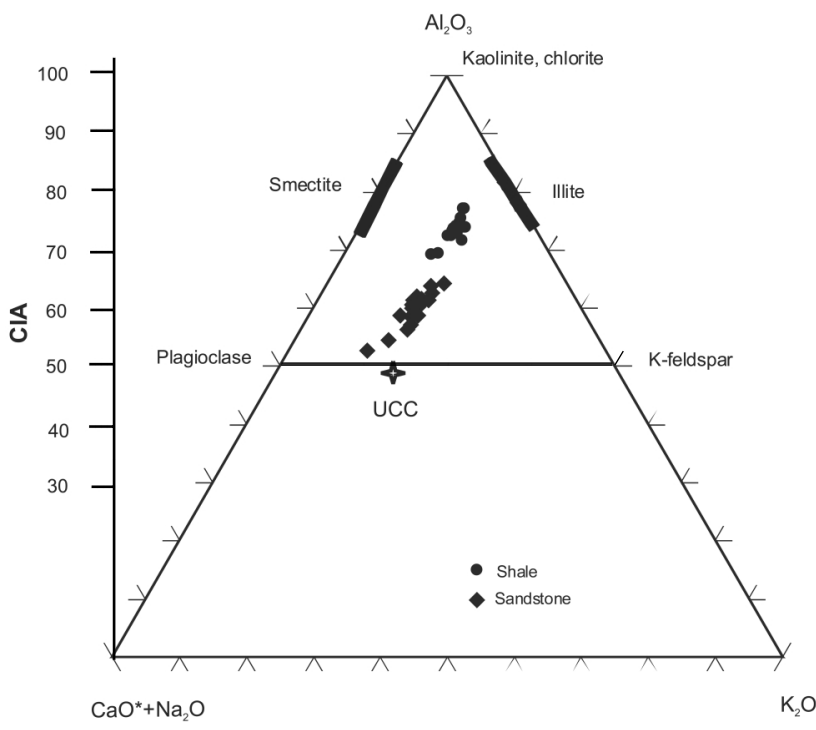

Fig. 13. CIA ternary diagram, $\mathrm{Al}_{2} \mathrm{O}_{3}-\mathrm{CaO}^{*}+\mathrm{Na}_{2} \mathrm{O}-\mathrm{K}_{2} \mathrm{O}$ (after Nesbitt and Young, 1982); $\mathrm{CaO}^{*}=\mathrm{CaO}$ in silicate phase.

weathering in the source areas whereas low values (i.e., 50 or less) indicate unweathered source areas. CIA values for the Surma Group sandstones vary from 53 to 65 , with an average 59 indicating the Surma sandstones were likely derived from the least weathered zones of soil profiles. The average CIA value (59) is a little above than
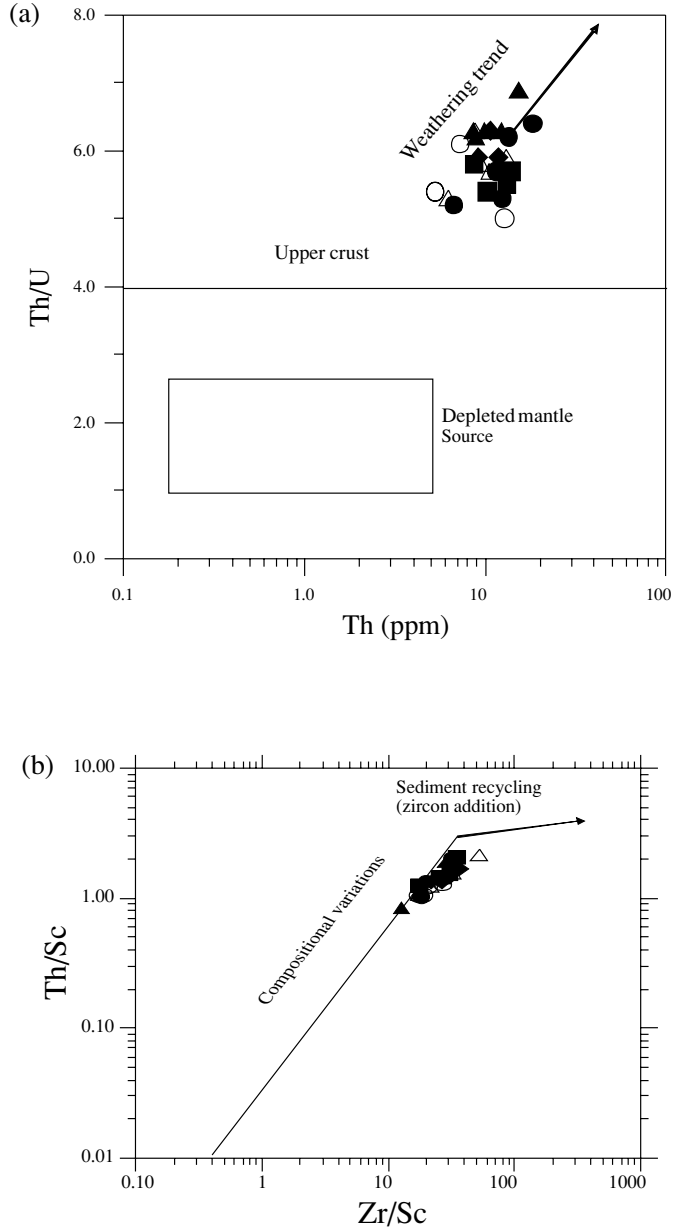

Fig. 14. Plots of (a) Th/U versus Th and (b) Th/Sc versus $\mathrm{Zr} / \mathrm{Sc}$ for the Surma Group sandstones (after McLennan et al., 1993).

that of the CIA value (50) of UCC. The calculated CIA values for the Surma Group sandstones are very similar to the CIA values of Yamuna River System sediments in the Himalaya ( 51 to 69; average $\sim 60$ ) (Dalai et al., 2002). The CIA values are also plotted in $\mathrm{Al}_{2} \mathrm{O}_{3}-\left(\mathrm{CaO} *+\mathrm{Na}_{2} \mathrm{O}\right)$ $\mathrm{K}_{2} \mathrm{O}$ (A-CN-K) diagram (Fig. 13). In the $\mathrm{A}-\mathrm{CN}-\mathrm{K}$ diagram the Surma Group sandstones plot a little away from the plagioclase-K-feldspar join line, indicating low to moderate chemical weathering of the source. Interbedded shales from the Surma Group show higher CIA values ( 70-78) (Rahman and Faupl, 2003b) comparable to and a little higher than average shale CIA $=70-75$, (Nesbitt and Young, 1982). The presence of plagioclase $(\sim 9 \%)$ in the shales (Rahman and Faupl, 2003b) may imply insignificant chemical weathering during sedimentation in the basin (Einsele, 1992, p. 364), but the CIA values of shales are clearly indicative of significant weathering in their source. The occurrence of abundant quartz, and small amounts of feldspar of the sandstones as well 
as illite and chlorite rich clay assemblages of the shales (Rahman and Faupl, 2003a, b) implies that the minerals are predominantly detrital and reflect the character of their source material (after Weaver, 1958). The thick pile of interbedded sandstones and mudrocks may have been resulted from rapid erosion of fast rising orogens. Integrating the CIA values of both sandstones and mudrocks, it is most likely that the sediments of the Miocene Surma Group were derived from different zones of weathering in the source soil profiles.

$\mathrm{Th} / \mathrm{U}$ in sedimentary rocks is of interest, as weathering and recycling typically result in loss of $\mathrm{U}$, leading to an elevation in the $\mathrm{Th} / \mathrm{U}$ ratio. The $\mathrm{Th} / \mathrm{U}$ ratio in most upper crustal rocks is typically between 3.5 and 4.0 (McLennan et al., 1993). In sedimentary rocks, Th/U values higher than 4.0 may indicate intense weathering in source areas or sediment recycling. $\mathrm{Th} / \mathrm{U}$ ratios in the Surma Group sandstones range from 5.0 to 6.9, with an average of 5.9 , indicating the derivation of these sediments from recycling of the crust. A Th/U versus Th plot for the Miocene Surma Group sandstones (Fig. 14a) shows a typical distribution similar to the average values of fine-grained sedimentary rocks reported by Taylor and McLennan (1985) and follows the normal weathering trend (McLennan et al., 1993). Sedimentary sorting and recycling can be monitored by a plot of $\mathrm{Th} / \mathrm{Sc}$ against $\mathrm{Zr} /$ Sc (McLennan et al., 1993). First-order sediments show a simple positive correlation between these ratios, whereas recycled sediments show a substantial increase in $\mathrm{Zr} / \mathrm{Sc}$ with far less increase in $\mathrm{Th} / \mathrm{Sc}$. The trend of increased $\mathrm{Zr} /$ Sc and almost constant $\mathrm{Th} / \mathrm{Sc}$ can also be exhibited by first-cycle sediments if they are derived from largely plutonic sources, as described by Roser and Korsch (1999) in the case of the Torlesse terrane of New Zealand. On the $\mathrm{Th} / \mathrm{Sc}$ versus $\mathrm{Zr} / \mathrm{Sc}$ diagram, the Surma Group sandstones follow a general trend consistent with their direct derivation from igneous rocks (Fig. 14b). The $\mathrm{Rb} /$ $\mathrm{Sr}$ ratios of sediments also monitor the degree of the source-rock weathering (McLennan et al., 1993). The Surma sandstones have average ratios of 0.50 , higher than that of the average upper continental crust $(0.32)$ but significantly lower than the average post-Archean Australian shale (0.80; McLennan et al., 1983). However, the Neogene shales have higher $\mathrm{Rb} / \mathrm{Sr}$ ratios $(\sim 1.3)$ than that of PAAS. The $\mathrm{Rb} / \mathrm{Sr}$ ratios of the Surma Group sediments (sandstones and mudrocks) therefore indicate least to significant weathering in the source area soil profiles.

\section{CONClusions}

The Miocene Surma Group sandstones have dominantly quartzolithic composition and a more quartzose one although some feldspathic and quartz arenitic sandstones are also recognized. The Surma sandstones contain abundant low-grade metamorphic, sedimentary lithics, low feldspar and low volcanic detritus, indicating that the sands were derived from a quartzose recycled orogen province.

The Surma Group sandstones have moderate to high $\mathrm{SiO}_{2}$ contents. Compared to the average sandstone value proposed by Turekian and Wedepohl (1961), they are depleted in $\mathrm{CaO}$ and enriched in $\mathrm{Al}_{2} \mathrm{O}_{3}$ and $\mathrm{MgO}$. Tectonic discrimination diagrams suggest an active continental margin to passive margin setting for the Surma Group sandstones.

$\mathrm{Eu} / \mathrm{Eu}^{*},(\mathrm{La} / \mathrm{Lu}) \mathrm{cn}, \mathrm{La} / \mathrm{Sc}, \mathrm{Th} / \mathrm{Sc}, \mathrm{La} / \mathrm{Co}, \mathrm{Th} / \mathrm{Co}$ and $\mathrm{Cr} / \mathrm{Th}$ ratios and the REE patterns indicate derivation of these sandstones from felsic source rocks. The existence of huge complexes of mafic/ultramafic rocks in the source region is most unlikely. CIA values for the Miocene Surma Group sandstones vary from 53 to 65 with an average 59 , indicating derivation from the least weathered zones of soil profiles. The average CIA value (59) is a little above than that of the CIA value (50) of upper continental crust. Shales from the Surma Group show higher CIA ( 70-78) values, indicating significant weathering in the source. Integrating the CIA values of the mudrocks, it is most likely that the sediments of the Miocene Surma Group were derived from the different zones of weathering in the source soil profiles, and likely from the rapid erosion of fast rising recycled orogens.

The geochemical characteristics preserve the signatures of a recycled provenance for the Miocene Surma Group sediments, in good agreement with framework mineralogy.

Acknowledgments - This research was carried out by the first author as a part of a Post-doctoral project (2003-2005) financed by JSPS (Japan Society for the Promotion of Science) Grant No. 15-03211. We are grateful to the reviewers Dr. Hakuyu Okada and Dr. Barry Roser for their constructive comments and suggestions to improve our manuscript.

\section{REFERENCES}

Alam, M. (1989) Geology and depositional history of Cenozoic sediments of the Bengal Basin of Bangladesh. Palaeogeogr., Palaeoclimatol., Palaeoecol. 69, 125-139.

Alam, M., Alam, M. M., Curray, J. R., Chowdhury, M. L. R. and Gani, M. R. (2003) An overview of the sedimentary geology of the Bengal Basin in relation to the regional tectonic framework and basin-fill history. Sedimentary geology of the Bengal Basin, Bangladesh, in relation to the AsiaGreater India collision and the evolution of the eastern Bay of Bengal. Sediment. Geol. 155 (Alam, M. M. and Curray, J. R., eds.), 179-208, Elsevier Science.

Bhatia, M. R. (1983) Plate tectonics and geochemical composition of sandstones. J. Geol. 91, 611-627.

Bhatia, M. R. and Crook, K. A. W. (1986) Trace element characteristics of graywackes and tectonic setting discrimina- 
tion of sedimentary basins. Contrib. Mineral. Petrol. 92, 181-193.

Crook, K. A. W. (1974) Lithogenesis and geotectonics: the significance of compositional variation in flysch arenites (graywackes). Soc. Econ. Paleontol. Mineral. Spec. Pub. 19, 304-310.

Cullers, R. L. (1994) The controls on the major and trace element variation of shales, siltstones, and sandstones of Pennsylvanian-Permian age from uplifted continental blocks in Colorado to platform sediment in Kansas, USA. Geochim. Cosmochim. Acta 58, 4955-4972.

Cullers, R. L. (2000) The geochemistry of shales, siltstones and sandstones of Pennsylvanian-Permian age, Colorado, USA: Implications for provenance and metamorphic studies. Lithos 51, 181-203.

Cullers, R. L. and Podkovyrov, V. N. (2000) Geochemistry of the Mesoproterozoic Lakhanda shales in southeastern Yakutia, Russia: Implications for mineralogical and provenance control, and recycling. Precamb. Res. 104, 77-93.

Cullers, R. L., Basu, A. and Suttner, L. J. (1988) Geochemical signature of provenance in sand-size material in soils and stream sediments near the Tobacco Root batholith, Montana, USA. Chem. Geol. 70, 335-348.

Curray, J. R. (1991) Geological history of the Bengal geosyncline. J. Assoc. Explor. Geophys. XII, 209-219.

Curray, J. R. and Munasinghe, T. (1991) Origin of the Rajmahal Traps and the $85 \mathrm{E}$ Ridge preliminary reconstructions of the trace of the Crozet hotspot. Geology 19, 1237-1240.

Curray, J. R., Emmel, F. J., Moore, D. G. and Reitt, R. W. (1982). Structure, tectonics and geological history of the northeastern Indian Ocean. The oceanic Basins and Margins. The Indian Ocean 6 (Nairn, A. E. M. and Stehli, F. G., eds.), 399-450, Plenum.

Dabard, M. P. (1990) Lower Brioverian formations (Upper Proterozoic) of the Armorican Massif (France): Geodynamic evolution of source areas revealed by sandstone petrography and geochemistry. Sediment. Geol. 69, 45-58.

Dalai, T. K., Krishnaswami, S. and Sarin, M. M. (2002) Major ion chemistry in the headwaters of the Yamuna river system: Chemical weathering, its temperature dependence and $\mathrm{CO}_{2}$ consumption in the Himalaya. Geochim. Cosmochim. Acta 66, 3397-3416.

Dickinson, W. R. (1985) Interpreting provenance relations from detrital modes of sandstones. Provenance of Arenites (Zuffa, G. G., ed.), 333-361, Advanced Study Institute Series 148, NATO.

Einsele, G. (1992) Sedimentary Basins. Springer Verlag, Berlin, $628 \mathrm{pp}$.

Evans, P. (1964) Tectonic framework of Assam. J. Geophys. Soc. India 5, 80-96.

Fairbridge, R. W. (ed.) (1972) The Encyclopedia of Geochemistry and Environmental Sciences. Van Nostrand Reinhold Company, New York, 1344 pp.

Feng, R. and Kerrich, R. (1990) Geochemistry of fine-grained clastic sediments in the Archean Abitibi greenstone belt, Canada: Implications for provenance and tectonic setting: Geochim. Cosmochim. Acta 54, 1061-1081.

France-Lanord, C., Derry, L. and Michard, A. (1993) Evolution of the Himalaya since Miocene time: isotopic and sedimentologic evidence from the Bengal Fan. Himalayan Tectonics (Treloar, P. J. and Searle, M., eds.), 603-621, Geol. Soc. London, Spec. Pub. 74.

Garver, J. I., Royce, P. R. and Smick, T. A. (1996) Chromium and nickel in shale of the Taconic foreland: a case study for the provenance of fine-grained sediments with an ultramafic source. J. Sediment. Res. 100, 100-106.

Hayashi, Ken-I., Fujisawa, H., Holland, H. D. and Ohmoto, H. (1997) Geochemistry of $1.9 \mathrm{Ga}$ sedimentary rocks from northeastern Labrador, Canada. Geochim. Cosmochim. Acta 61, 4115-4137.

Herron, M. M. (1986) Geochemical classification of terrigeneous sands and shales from core or log data. J. Sediment. Petrol. 58, 820-829.

Hiscott, R. N. (1984) Ophiolitic source rocks for Taconic-age flysch: Trace element evidence. Geol. Soc. Am. Bull. 95, 1261-1267.

Holland, H. D. (1978) The Chemistry of the Atmosphere and Oceans. Wiley, New York, 351 pp.

Ingersoll, R. V., Bullard, T. F., Ford, R. L., Grimm, J. P., Pickle, J. D. and Sares, S. W. (1984) The effect of grain size on detrital modes: a test of the Gazzi-Dickinson point counting method. J. Sediment. Petrol. 54, 103-116.

Maynard, J. B., Valloni, R. and Yu, H. S. (1982) Composition of modern deep-sea sands from arc-related basins. TrenchForearc Geology: Sedimentation and Tectonics on Modern and Ancient Active Plate Margins (Legget, J. K., ed.), 2140, Geol. Soc. Am. Spec. Pap. 284.

McCann, T. (1991) Petrological and geochemical determination of provenance in the southern Welsh Basin. Developments in Sedimentary Provenance Studies (Morton, A. C., Todd, S. P. and Haughton, P. D. W., eds.), 215-230, Geol. Soc. Spec. Pub. 57.

McLennan, S. M. (2001) Relationships between the trace element composition of sedimentary rocks and upper continental crust. Geochemistry Geophysics Geosystems 2, paper number 2000GC000109 (8994 words, 10 figures, 5 tables). Published April 20, 2001.

McLennan, S. M., Taylor, S. R. and Eriksson, K. A. (1983) Geochemistry of Archean shales from the Pilbara Supergroup, Western Australia. Geochim. Cosmochim. Acta 47, 1211-1222.

McLennan, S. M., Hemming, S., McDaniel, D. K. and Hanson, G. N. (1993) Geochemical approaches to sedimentation, provenance, and tectonics. Processes Controlling the Composition of Clastic Sediments (Johnson, M. J. and Basu, A., eds.), 21-40, Geol. Soc. Am. Spec. Pap. 284.

Mitchell, A. H. G. and Reading, H. G. (1986) Sedimentation and tectonics. Sedimentary Environments and Facies (Reading, H. G., ed.), 471-519, Blackwell Scientific Publications.

Nesbitt, H. W. and Young, G. M. (1982) Early Proterozoic climates and plate motions inferred from major element chemistry of lutites. Nature 299, 715-717.

Nesbitt, H. W. and Young, G. M. (1996) Petrogenesis of sediments in the absence of chemical weathering: effects of abrasion and sorting on bulk composition and mineralogy. Sedimentology 43, 341-358.

Osman, M. (1996) Recent to Quaternary River Nile Sediments: A sedimentological characterization on samples from As- 
wan to Naga-Hammadi, Egypt. Unpub. Ph.D. thesis, Univ. of Vienna, Vienna, $162 \mathrm{pp}$.

Potter, P. E. (1978) Petrology and chemistry of modern big river sands. J. Geol. 86, 423-449.

Rahman, M. J. J. (1999) Sandstone Petrography of the subsurface Neogene Surma Group of the Sylhet Trough, Bengal Basin. Bangladesh Geoscience Journal 5, 13-30.

Rahman, M. J. J. and Faupl, P. (2003a) ${ }^{40} \mathrm{Ar} /{ }^{39} \mathrm{Ar}$ multigrain dating of detrital white mica of sandstones of the Surma Group in the Sylhet Trough, Bengal Basin, Bangladesh. Sedimentary geology of the Bengal Basin, Bangladesh, in relation to the Asia-Greater India collision and the evolution of the eastern Bay of Bengal. Sediment. Geol. 155 (Alam, M. M. and Curray, J. R., eds.), 383-392, Elsevier Science.

Rahman, M. J. J. and Faupl, P. (2003b) The composition of the subsurface Neogene shales of the Surma Group from the Sylhet Trough, Bengal Basin, Bangladesh. Sedimentary geology of the Bengal Basin, Bangladesh, in relation to the Asia-Greater India collision and the evolution of the eastern Bay of Bengal. Sediment. Geol. 155 (Alam, M. M. and Curray, J. R. eds.), 407-417, Elsevier Science.

Roser, B. P. and Korsch, R. J. (1986) Determination of tectonic setting of sandstone-mudstone suites using $\mathrm{SiO}_{2}$ content and $\mathrm{K}_{2} \mathrm{O} / \mathrm{Na}_{2} \mathrm{O}$ ratio. J. Geol. 94, 635-650.

Roser, B. P. and Korsch, R. J. (1988) Provenance signatures of sandstone-mudstone suites determined using discriminant function analysis of major-element data. Chem. Geol. 67, 119-139.

Roser, B. P. and Korsch, R. J. (1999) Geochemical characterization, evolution and source of a Mesozoic accretionary wedge: The Torlesse terrane, New Zealand. Geol. Mag. 136, 493-512.

Salt, C. A., Alam, M. M. and Hossain, M. M. (1986) Bengal Basin: current exploration of the hinge zone area of southwestern Bangladesh. Proc. 6th Offshore Southwest Asia Conference, 55-57.

Shaw, D. M. (1968) A review of K-Rb fractionation trends by covariance analysis. Geochim. Cosmochim. Acta 32, 573602.

Taylor, S. R. and McLennan, S. M. (1985) The Continental Crust; Its Composition and Evolution. Blackwell, London, $312 \mathrm{pp}$.

Turekian, K. K. and Wedepohl, K. H. (1961) Distribution of the elements in some major units of the earth's crust. Geol. Soc. Am. Bull. 72, 175-192.

Uddin, A. and Lundberg, N. (1998) Unroofing history of the eastern Himalaya and the Indo-Burman Ranges: Heavymineral study of Cenozoic sediments from the Bengal Basin, Bangladesh. J. Sediment. Res. 68, 465-472.

Uddin, A. and Lundberg, N. (1999) A paleo-Brahmaputra? Subsurface lithofacies analysis of Miocene deltaic sediments in the Himalayan-Bengal system, Bangladesh. Sediment. Geol. 123, 239-254

Weaver, C. E. (1958) Geologic interpretation of argillaceous sediments. Part 1. Origin and Significance of clay minerals in sedimentary rocks. Am. Ass. Petrol. Geologists Bull. 42, 254-271.

Wronkiewicz, D. J. and Condie, K. C. (1989) Geochemistry and provenance of sediments from the Pongola Supergroup, South Africa: Evidence for a 3.0-Ga-old continental craton. Geochim. Cosmochim. Acta 53, 1537-1549. 